\title{
1 Characterisation of a mitochondrial iron transporter of the pathogen Trypanosoma
}

\section{2 brucei}

3 Fuli Zheng ${ }^{1,3 \#}$, Claudia Colasante ${ }^{2 \#}$, Frank Voncken ${ }^{3 *}$

$4{ }^{1}$ Department of Preventive Medicine, School of Public Health, Fujian Medical University,

5 Xueyuan Road, Fujian, 350122, P.R. China

6 '2Institute for Anatomy and Cell Biology, Division of Medical Cell Biology, Aulweg 123,

7 University of Giessen, 35392 Giessen, Germany

$8{ }^{3}$ Department of Biomedical Sciences, School of Life Sciences, University of Hull, Cottingham

9 Road, Hull, HU6 7RX, United Kingdom

10 \# These authors contributed equally to the paper

11

12 Running Title: The T. brucei mitochondrial iron transporter TbMCP17

13 *To whom correspondence should be addressed: Claudia.Colasante@anatomie.med.uni-

14 giessen.de

15 Keywords: mitochondrial transport, iron metabolism, Trypanosoma brucei, mitochondrial

16 carrier family-proteins

17

18

C2019, Elsevier. This manuscript version is made available under the CC-BY-NC-ND 4.0 license http:// creativecommons.org/licenses/by-nc-nd/4.0/ 


\section{ABSTRACT}

20 Similar to higher eukaryotes, the protist parasite $T$. brucei harbours several iron-containing

21 proteins that regulate DNA and protein processing, oxidative stress defence and mitochondrial

22 respiration. The synthesis of these proteins occurs either in the cytoplasm or within the

23 mitochondrion. For mitochondrial iron cluster protein synthesis iron needs to be transported

24 across the solute impermeable mitochondrial membrane. In T. brucei we previously identified

2524 mitochondrial carrier proteins (TbMCPs) sharing conserved structural and functional

26 features with those from higher eukaryotes. One of these carriers (TbMCP17) displayed high

27 similarity with the iron carriers MRS3, MRS4 from yeast and mitoferrin from mammals,

28 insects and plants. In the present study we demonstrated that $T b \mathrm{MCP} 17$ functions as an iron

29 carrier by complementation studies using MRS3/4-deficient yeast. Depletion of TbMCP17 in

30 procyclic form $T$. brucei resulted in growth deficiency, increased sensitivity to iron

31 deprivation, and lowered mitochondrial iron content. Taken together our results suggest that

$32 \mathrm{TbMCP} 17$ functions as a mitochondrial iron transporter in the parasite T. brucei. 


\section{INTRODUCTION}

35 Trypanosoma brucei is a protist parasite belonging to the class of the Kinetoplastida and 36 causing African sleeping sickness in humans and Nagana in cattle [1-3]. During its life cycle,

37 the parasite is transferred from the insect vector, the tsetse fly, to the mammalian host thereby 38 undergoing substantial remodelling in morphology and metabolism [4]. T. brucei replicative 39 stages that can be cultured in vitro are the bloodstream form (BSF) found in the blood and 40 cerebrospinal fluid of mammals, and the procyclic form (PCF) residing in the mid-gut of the 41 tsetse fly. The BSF, which lives in a glucose rich environment, derives its energy exclusively 42 through substrate level phosphorylation reactions during glycolysis that is partially located 43 within specialised peroxisomes (glycosomes) [5,6]. Within the insect vector, PCF T. brucei 44 uses glucose and the amino acid proline to generate ATP through glycolysis and mitochondrial 45 respiration $[5,7-11]$.

46 As the energy metabolism, the mechanism by which iron and heme are taken up is adapted to 47 iron availability within the hosts and differs between BSF and PCF. The BSF acquires 48 transferrin-bound iron and heme in the form of a haptoglobin-haemoglobin complex through 49 receptor mediated endocytosis directed to the endo-lysosomal system [12-15]. Due to the 50 absence of transferrin in the insect gut, the PCF takes up ferrous iron by endocytosis of reduced 51 ferric complexes [16] while heme is acquired through the membrane transporter $\mathrm{TbHrg}$ [15].

52 Once within the cytoplasm, iron and heme are redistributed to intracellular sites for the 53 synthesis of iron-containing protein. Like higher eukaryotes, T. brucei incorporates iron into $54 \mathrm{iron} /$ sulphur clusters $(\mathrm{Fe} / \mathrm{S})$ or as non-heme and non-Fe/S iron into enzymes involved in 55 cellular processes such as DNA synthesis, protein translation, oxidative stress defence and 56 cytochrome respiration [12,14,17]. Iron-dependent enzymes that have been identified in $T$. 57 brucei are, for example, the ribonucleotide reductase that catalyses the reduction of 58 ribonucleotides to deoxyribonucleotides necessary for DNA synthesis $[18,19]$, superoxide 
59 dismutase, which catalyses the dismutation of the superoxide radical $\left(\mathrm{O}_{2}{ }^{\circ}\right)$ into hydrogen

60 peroxide [20], alternative oxidase, which re-oxidises glycolysis derived NADH [21-23],

61 aconitase and fumarase, both TCA cycle enzymes [24] or the cytochromes of the respiratory

62 chain [25]. Machineries responsible for the mitochondrial and cytosolic iron-sulphur cluster

63 protein assembly and for the mitochondrial transfer of iron to non-heme/non-FeS iron proteins

64 have all been identified in T. brucei $[12,26]$. The mitochondrion is an indispensable site for

65 the synthesis of iron-sulphur cluster proteins involved in electron transport during respiration,

66 enzymatic catalysis, oxidative stress response and the regulation of mitochondrial iron

67 homoeostasis [27]. For the assembly of these iron-containing proteins, iron has to cross the

68 solute-impermeable inner mitochondrial membrane. In yeast and vertebrates iron is imported

69 into the mitochondrion by the mitochondrial iron transporters MRS3 and 4 [28-30], and

70 mitoferrin, respectively [31]. These transporters all belong to the mitochondrial carrier family

71 (MCF, SLC25A), which exchange various solutes, including carboxylates, nucleotides,

72 inorganic phosphate ornithine, carnitine, and glutamine across the mitochondrial inner

73 membrane [32,33]. MCF proteins (MCPs) control the influx rate of metabolic intermediates

74 into the mitochondrion, regulate the flux through metabolic processes and maintain the cellular

75 redox and ATP homeostasis [34]. The protein structure of MCPs is conserved and consist of

76 six transmembrane domains connected by short hydrophilic loops. Each odd numbered

77 transmembrane domain and hydrophilic loop contains a conserved signature motif [33] and

78 amino acid contact points, which determine the substrate specificity of the carrier subtype [35].

79 We previously reported that $T$. brucei possesses 24 MCPs (TbMCPs) containing conserved

80 structural and sequence features of MCPs from higher eukaryotes [36-39]. Amongst these

81 carriers we identified one with high similarity to MRS3, MRS4 and mitoferrin and named it

$82 T b \mathrm{MCP} 17$ [36]. In the present study, sequence alignments and phylogenetic reconstruction

83 revealed the close relationship of $T b \mathrm{MCP} 17$ to plant and mammalian iron carriers. The 
84 functionality of $T b \mathrm{MCP} 17$ as an iron carrier was confirmed by complementation studies using 85 MRS3/4-deficient yeast. We also show that in PCF, the removal of TbMCP17 caused a 86 significant growth defect, increased the sensitivity to iron deprivation, and lowered the 87 mitochondrial iron content. Taken together, our findings suggest that $T b \mathrm{MCP} 17$ functions as 88 a mitochondrial iron transporter in the parasite T. brucei.

89 


\section{Phylogenetic reconstruction and sequence analysis}

94 Multiple sequence alignments were generated using ClustalO [40,41]. Phylogenetic trees were

95 constructed using MEGA7 (Molecular Evolutionary Genetics Analysis version 7.0; [42].

96 Protein-Blast (blast.ncbi.nlm.nih.gov) was used to retrieve iron carrier protein sequences from

97 trypanosomatids, plants, insects, fungi and mammals. These were then imported into MEGA7

98 and aligned. Using the neighbour-joining (NJ) method [43], a NJ tree was drawn with bootstrap

99 set to 1000 .

100 The GenBank (gb), EMBL (emb), NCBI (XP) and Swiss protein (sp) accession numbers for

101 the TbMCP17 alignment and the phylogenetic reconstruction were as follows: TbMCP17,

102 Trypanosoma brucei brucei Tb927.3.2980; Trypanosoma brucei gambiense

103 emb_CBH09968.1; Trypanosoma congolense emb_CCC89759.1; Trypanosoma vivax

104 emb_CCC47046.1; Trypanosoma cruzi XP_822091.1; Trypanosoma vivax gb_EKG04497.1;

105 Trypanosoma rangeli gb_ESL10208.1; Leishmania amazonensis gb_ALP75642.1;

106 Leishmania mexicana emb_CBZ23750.1; Leishmania major emb_CBZ12619.1; Leptomonas

107 seymouri gb_KPI87731.1; Leishmania panamensis gb_AIO00226.1; Ustilago hordei

108 emb_CCF54811.1; Ustilago maydis gb_KIS71149.1; Pseudozyma brasiliensis

109 gb_EST06361.1; Rhizopus microspores emb_CEJ04189.1; Moesziomyces antarcticus

110 gb_ETS61106.1; Drosophila willistoni gb_EDW83029.1; Bactrocera cucurbitae

111 XP_011180944.1; Drosophila mojavensis gb_EDW14539.1; Gossypium raimondii

112 gb_KJB83905.1; Fragaria vesca XP_004294768.1; Madurella mycetomatis gb_KOP45184.1;

113 Phanerochaete carnosa gb_EKM49983.1; Gossypium arboreum gb_KHG15442.1;

114 Rhizoctonia solani emb_CUA78164.1; Drosophila virilis gb_EDW59237.2; Botrytis cinerea

115 XP_001553628.1; Sesamum indicum XP_011080718.1; Homo sapiens (MFRN1) 
116 gb_EAW63617.1; Homo sapiens (MFRN2) gb_AAK49519.1; Saccharomyces cerevisiae

117 (MRS3p), gb_EGA61827.1; Saccharomyces cerevisiae (MRS4p) gb_AJS43107.1;

118 Arabidopsis thaliana gb_AAP42736.1; Zea mays (MRS3) gb_ACG42379.1; Mus musculus

119 (MFRN1) gb_AAL23859.1; Mus musculus (MFRN2) gb_AAH25908.1; Bos taurus (MFRN1)

120 gb_AAI03256.1; Bos taurus (MFRN2) NP_001192481.1; Lucilia cuprina gb_KNC31521.1;

121 Drosophila busckii gb_ALC47563.1; Harpegnathos saltatorgb_EFN83637.1; Acyrthosiphon

122 pisum NP_001280444.1; Aspergillus niger XP_001390994.2.

\section{Culture and transfection of Trypanosoma brucei}

125 Procyclic form PCF449 were grown in a $27{ }^{\circ} \mathrm{C}$ incubator in MEM-PROS medium [24]

126 supplemented with $10 \%$ heat-inactivated foetal bovine serum (Lonza), $2.5 \mathrm{mg} / \mathrm{ml}$ of heme (in

$127100 \mathrm{mM} \mathrm{NaOH}$ ) $1 \%$ of penicillin/streptomycin solution (Sigma) and $5 \mathrm{mM}$ proline (Sigma).

128 For the experiments described in this paper Trypanosoma brucei cell lines were transfected

129 with different plasmids and clonal cell lines were selected using antibiotics according to the 130 previously published protocol [44].

131

\section{Yeast functional complementation}

133 Saccharomyces cerevisiae strains used in this study were wildtype strain BY4741 (MATa

134 his $3 \Delta 1$ leu $2 \Delta 0$ met $15 \Delta 0$ ura $3 \Delta 0$, referred to as wild type below) and mitochondrial iron carrier 135 (MRS3/4) deficient strain GW403 (mrs3/4A; MATa his3- $\Delta 1$ leu2-3 leu2-112 ura3-52 trp1-

$136289 \operatorname{mrs} 3 \Delta:: \operatorname{loxP} \operatorname{mrs} 4 \Delta:: \operatorname{loxP}$; referred to as $\Delta \mathrm{MRS} 3 / 4$ below) [45]. Yeast strains were

137 maintained on standard YPD medium. For clone-selection following transfection, synthetic

138 complete (SC) medium without uracil $(0.67 \%$ yeast nitrogen base without amino acids, $1.4 \%$

139 drop-out medium supplements without histidine, leucine, tryptophan and uracil) supplemented

140 with $60 \mathrm{mg} / \mathrm{L}$ of leucine, $20 \mathrm{mg} / \mathrm{L}$ of tryptophan, $20 \mathrm{mg} / \mathrm{L}$ of histidine, $2 \%$ of agar and $2 \%$ of 
141 dextrose was used. To test cell growth on different carbon sources, YPD and YPG media were

142 used. For plate growth experiments, SC medium was supplemented with dextrose (SCD

143 medium) or glycerol (SCG medium). To simulate iron deprived condition, $80 \mu \mathrm{M}$ of iron

144 chelator bathophenanthrolinedisulfonic acid (BPS, Sigma) was added to the SC medium.

145 The complete open reading frames of TbMCP17 and S. cerevisiae MRS3 (YJL133W) were

146 PCR-amplified using the primer combination 5'-gaGGATCCatggtttccgagggcacttccgctg-3'/5'-

147 cgAAGCTTttaccgttcctccatgaacttcttggc-3', and 5'-gaGGATCCatggtagaaaactcgtcgagtaata-

148 3'/5'-cgCTGCAGctaatacgtcattaggaaatgttttgcacattc -3', respectively. Restriction enzyme sites

149 used for subsequent cloning into the yeast centromeric expression vector pCM190 (Euroscarf)

150 are underlined and capitalised.

151 Plasmids containing either TbMCP17 or MRS3 were transformed into the yeast strain GW403,

152 using the lithium acetate/single-stranded carrier DNA method described by Gietz and Woods

153 [46]. Obtained yeast clones were maintained on synthetic complete dextrose (glucose) medium

154 without uracil in the presence of tetracycline. Heterologous protein expression in yeast was

155 induced by tetracycline removal [47].

156 For the growth experiments, yeast cells from an overnight starter culture were inoculated in

$15720 \mathrm{ml}$ of medium and the $\mathrm{OD}_{600}$ measured every 3 to $4 \mathrm{~h}$ until cells reached stationary phase.

158 For plating experiment, yeast was spotted on agar plates at $\mathrm{OD}_{600} 1,0.1,0.01$ and 0.001 .

160 Over-expression of TbMCP17

161 The open reading frame (ORF) of TbMCP17 was amplified from T. brucei 449 genomic DNA 162 using the primer pair 5'-ggacggAAGCTTaccatggtttccgagggcacttccgctg-3'/5'-

163 gcttgcaGGATCCccgttcctccatgaacttcttggc-3'. The restriction sites used for subsequent cloning 164 into the pHU1 or pHU2 T. brucei expression vectors [48] are underlined and capitalised. The 165 expression vectors generate either C-terminally (pHU1) or N-terminally (pHU2) $2 \times$ myc- 
166 tagged recombinant proteins. Comparison of the cloned TbMCP17 sequence with the sequence

167 obtained from the genome sequence database (GeneDB) of T. brucei strain 927 revealed only

168 a few sequence differences at the DNA level, but none in the predicted amino acid sequence.

169 The obtained vectors were used to transfect the PCF Trypanosoma brucei strain Lister 427,

170 stably expressing the tetracycline (tet) repressor from the plasmid pHD449 (PCF449).

171 Hygromycin resistant clonal cell lines were isolated and analysed by western blotting after

172 induction of tagged $T b \mathrm{MCP} 17$ expression using tetracycline $(0.5 \mathrm{mg} / \mathrm{ml})$. The generated cell

173 lines are further referred to as $T b \mathrm{MCP} 17-\mathrm{cmyc}^{\mathrm{ti}}$ (C-terminally-tagged $\left.T b \mathrm{MCP} 17\right)$ and

$174 T b \mathrm{MCP} 17-\mathrm{nmyc}^{\mathrm{ti}}$ (N-terminally-tagged TbMCP17).

175

176 Immunofluorescence analysis

177 Immunofluorescence analysis using paraformaldehyde-fixed trypanosomes was performed as

178 previously described [36] with minor adjustments: an aliquot of culture containing

179 approximately $1 \times 10^{7}$ cells was stained with $0.5 \mu \mathrm{M}$ Mitotracker Red CMXRos (Sigma) for 30

180 min. Cells were then fixed onto coverslips using freshly made 4\% (w/v) paraformaldehyde in

181 1x PBS and left to attach overnight at $4{ }^{\circ} \mathrm{C}$. For permeabilisation $0.2 \%(\mathrm{w} / \mathrm{v})$ Triton X-100 was

182 added followed by incubation with $0.5 \%(\mathrm{w} / \mathrm{v})$ gelatine in 1x PBS. Afterwards, myc-primary

183 antibody (Roche) was added to the coverslips at a dilution of 1:500 in $0.5 \%(\mathrm{w} / \mathrm{v})$ gelatine in

184 1x PBS and incubated for 60 min. Coverslips were then washed twice in 1x PBS. A 1:500

185 dilution of the secondary antibody in $0.5 \%(\mathrm{w} / \mathrm{v})$ gelatine in 1x PBS (Alexa Fluor 488 goat

186 anti-mouse, Thermofisher) was then added and incubated for $60 \mathrm{~min}$ in the dark. The coverslip

187 was placed on a microscope slide coated with mounting medium containing DAPI (Thermo).

188 Slides were stored in the dark at $4^{\circ} \mathrm{C}$ and analysed using a laser scanning confocal microscope

189 (Zeiss) within 2-3 days.

190 
191 Generation of the conditional TbMCP17 double-knock out cell line

192 The conditional double knock out of TbMCP17 was constructed using the target gene

193 replacement method $[39,49,50]$. The cell line $T b \mathrm{MCP} 17-\mathrm{cmyc}^{\mathrm{ti}}$ was used as parental cell line

194 for the generation of the conditional TbMCP17 double-knock out cell line. The 5'-UTR and

195 3'-UTR of TbMCP17 were PCR amplified using the primer pairs 5'-

196 gctaGAGCTCcgtgtcgtgaggtggagaggtgatg-3'/5'-

197 gctaACTAGTcacacatcaccgcagccaagcaaaacaacg-3' (5'-UTR 475 bp fragment), and 5'-

198 gcatGGATCCccgtgttcttgtttcaggtgtgaacc-3'/5' -ctatGGGCCCgtcaaacacattactggagcgg-3' for

199 the (3'-UTR $484 \mathrm{bp}$ fragment). Using the underlined and capitalised restriction enzyme sites

200 the amplified fragments were inserted on either side of the NEO (G418) (NEO-TbMCP17-

201 knock out vector) and BSD (blasticidin) (BSD-TbMCP17-knock out vector) antibiotic

202 resistance cassettes, bearing actin 5'-splice sites and actin 3'-UTR [50]. After transfection of

203 the TbMCP17-cmycti cell line with the NEO-TbMCP17-knock out construct and clonal

204 selection with $15 \mu \mathrm{g} / \mathrm{ml}$ G418, the single-knock out cell line

$205 \Delta$ TbMCP17::NEO/TbMCP17/TbMCP17-cmyc ${ }^{\text {ti }}$ was obtained. The double knock out cell line

$206 \triangle T b M C P 17:: \mathrm{NEO} / \triangle T b M C P 17:: \mathrm{BSD} / T b M C P 17-\mathrm{cmyc}^{\mathrm{ti}}$, (further referred to as

$207 \triangle T b M C P 17 / T b M C P 17-\mathrm{cmyc}^{\mathrm{ti}}$ in this paper), was obtained after transfection of the

$208 \Delta T b M C P 17:: \mathrm{NEO} / T b M C P 17 / T b M C P 17-\mathrm{cmyc}^{\text {ti }}$ cell line using the BSD-TbMCP17-knock out

209 plasmid and clonal selection with $15 \mu \mathrm{g} / \mathrm{ml} \mathrm{G} 418$ and $10 \mu \mathrm{g} / \mathrm{ml}$ blasticidin. The TbMCP17

210 single- and double-knock out cell lines were cultured in the presence of tetracycline $(1 \mu \mathrm{g} / \mathrm{ml})$

211 to maintain $T b \mathrm{MCP} 17-\mathrm{cmyc}$ expression and ensure cell viability. The deletion of TbMCP17

212 was confirmed by PCR analysis.

214 Depletion of $\mathbf{T b M C P 1 7}$ by RNA interference 
215 Inhibition of TbMCP17 expression was performed in PCF T. brucei using RNA interference 216 (RNAi) [51]. The primer combinations 5'-gAAGCTTccaccccatttgatgttatcaagcagc-3'/5'217 ggCTCGAGtgactaagacatagcgcaccgcatcgg-3' and 5'-cGGATCCccatttgatgttatcaagcagcggatg218 3'/5'-cAAGCTTtccCTCGAGcattgacagggcaccagcaggagcg-3' were used to PCR amplify the $219387 \mathrm{bp}$ sense and $467 \mathrm{bp}$ antisense sequences of $T b M C P 17$, respectively. The restriction 220 enzyme sites used for subsequent cloning into the vector pHD676 are underlined and 221 capitalised. The pHD676-TbMCP17 RNAi vector was used for transfection of procyclic form 222 T. brucei PCF449. The TbMCP17RNAi cell line was obtained after clonal selection using 223 hygromycin $(25 \mu \mathrm{g} / \mathrm{ml})$ and phleomycin $(0.5 \mu \mathrm{g} / \mathrm{ml})$.

\section{PCF $T$. brucei growth analysis}

226 For growth analysis, PCF cultures were diluted to a density of $5 \times 10^{5}$ cells $/ \mathrm{ml}$ at the start of the 227 experiments. During the growth experiments of the TbMCP17 overexpressing cells $228\left(T b M C P 17-\mathrm{cmyc}^{\mathrm{ti}}\right.$ and $\left.T b \mathrm{MCP} 17-\mathrm{nmyc}^{\mathrm{ti}}\right), 10 \mu \mathrm{g} / \mathrm{ml}$ tetracycline were added daily to maintain 229 expression of the recombinant myc-tagged proteins. During the growth experiments of cells 230 depleted of $T b M C P 17$ by conditional knock out ( $\left.\triangle T b M C P 17 / T b M C P 17-\mathrm{cmyc}^{\mathrm{ti}}\right)$ tetracycline 231 was removed from the cell culture $24 \mathrm{~h}$ prior the start of the growth experiments and cells were 232 thoroughly washed using MEM-Pros to stop the expression of TbMCP17-cmyc. During the 233 growth experiments of cells in which $T b \mathrm{MCP} 17$ was depleted by RNAi $10 \mu \mathrm{g} / \mathrm{ml}$ tetracycline 234 were added daily to induce and maintain the RNA interference. Cell densities were determined 235 every $24 \mathrm{~h}$ using a haemocytometer. In some experiments, the iron chelator deferoxamine 236 (Sigma) was added at different concentrations (10 $\mu \mathrm{M}$ to $100 \mu \mathrm{M})$ to obtain iron deprived 237 conditions.

\section{Western Blot}


239 For each SDS-PAGE lane $5 \times 10^{6}$ cells were pelleted, resuspended in denaturing SDS-

240 containing Laemmli buffer, and heated at $95^{\circ} \mathrm{C}$ for $5 \mathrm{~min}$. Proteins were separated on a $12 \%$

241 SDS-PAGE and then transferred to PVDF (GE Health Care Life Sciences) membranes at 100

$242 \mathrm{~V}$ for $50 \mathrm{~min}$ in Towbin buffer (48 mM Tris, $39 \mathrm{mM}$ Glycine, 20\% (v/v) methanol, $\mathrm{pH} 8.3$ ).

243 Membranes were blocked with 5\% w/v skimmed milk in Tris-buffered saline buffer (TBS)

244 and $0.1 \%$ Tween-20 (milk/TBST) at room temperature for $1 \mathrm{~h}$. Subsequently, membranes were

245 incubated with anti-c-myc (Roche) primary antibody diluted 1:2000 in milk/TBST, for $1 \mathrm{~h}$ at

246 room temperature. Membranes were then washed 3 times with excess TBST and incubated

247 with anti-mouse IgG HRP (Abcam) in secondary antibody diluted 1:2000 in milk/TBST, for

$2481 \mathrm{~h}$ at room temperature. Protein detection was performed using an ECL detection kit 249 (Amersham, GE Healthcare). Coomassie brilliant blue (CBB) staining was used as loading 250 control.

252 Iron measurement

253 Cells were grown in iron and heme depleted medium to avoid interference during iron 254 measurements. Depletion was achieved by pre-dialysing the foetal calf serum (FCS) using a 255 Slide-A-Lyzer ${ }^{\mathrm{TM}} \mathrm{G} 2$ dialysis cassette, $2 \mathrm{~K}$ molecular weight cut-off, $70 \mathrm{~mL}$ (Thermo) 256 according to manufacturer's protocol. Briefly, heat-inactivated FCS was added to the dialysis 257 cassette and put into $2 \mathrm{~L}$ of $1 \mathrm{x}$ PBS at $4^{\circ} \mathrm{C}$ for $3 \mathrm{~h}$. After this time, the $1 \mathrm{x}$ PBS was discarded, 258 replaced by fresh 1x PBS and dialysis was repeated 2 times more. Medium was then prepared 259 using the dialysed FCS and without the addition of heme.

260 Mitochondria of T. brucei were isolated by digitonin-fractionation according to previously 261 published protocols [52]. Cellular and mitochondrial iron content were measured using a 262 colorimetric ferrozine-based assay [53] with some modifications. Briefly, $100 \mu 1$ aliquots of 263 whole cell or mitochondrial lysates derived from $10^{8}$ trypanosomes were placed in Eppendorf 
264 tubes and mixed with $100 \mu \mathrm{l}$ of $10 \mathrm{mM} \mathrm{HCl}$, and $100 \mu \mathrm{l}$ iron-releasing reagent (a freshly mixed

265 solution of equal volumes of $1.4 \mathrm{M} \mathrm{HCl}$ and $\left.4.5 \%(\mathrm{w} / \mathrm{v}) \mathrm{KMnO}_{4}\right)$. The mixture was then 266 incubated for $2 \mathrm{~h}$ at $60{ }^{\circ} \mathrm{C}$ within a fume hood. After cooling to room temperature, $30 \mu \mathrm{l}$ of the 267 iron-detection reagent (6.5 $\mathrm{mM}$ ferrozine, $6.5 \mathrm{mM}$ neocuproine, $2.5 \mathrm{M}$ ammonium acetate, and $2681 \mathrm{M}$ ascorbic acid) were added to each tube. After $30 \mathrm{~min}$, the absorbtion was measured at $269550 \mathrm{~nm}$. The iron content of the sample was calculated by comparing its absorbtion to that of 270 a range of equally treated iron $\left(\mathrm{FeCl}_{3}\right)$ standards $(0-300 \mu \mathrm{M})$.

272 qRT PCR

273 One-step real time qPCR was used to determine the expression levels of $T b M C P 17$ after RNAi. 274 Total RNA was isolated from $1 \times 10^{7}$ PCF T. brucei using TriFast (PeqLab Biotechnology $275 \mathrm{GmbH}$ ) according to manufacturer's protocol. For cDNA synthesis $2 \mu \mathrm{g}$ total RNA in $10 \mu \mathrm{l}$ 276 RNase-free water were mixed with $4 \mu \mathrm{l}$ of $5 \times$ M-MuLV reaction buffer, $1 \mu 1$ of RiboLock 277 RNase inhibitor, $2 \mu \mathrm{l}$ of $10 \mathrm{mM}$ dNTP, and $2 \mu \mathrm{l}$ of M-MuLV reverse transcriptase (cDNA 278 synthesis kit, Thermo). The reaction was incubated at $25^{\circ} \mathrm{C}$ for $5 \mathrm{~min}$, followed by $60 \mathrm{~min}$ at $27937{ }^{\circ} \mathrm{C}$. Then the reaction was terminated by incubation at $70{ }^{\circ} \mathrm{C}$ for $5 \mathrm{~min}$. RT-PCR for the 280 detection of TbMPC17 was performed using $1 \mu 1$ of synthesised cDNA and the QuantiTect 281 SYBR Green RT-PCR kit (QIAGEN) to set up a $25 \mu 1$ PCR reaction. CT values of 282 experimental samples (in triplicates) were compared with wild type CT after normalising CT 283 from the house-keeping genes tubulin.

\section{TbMCP17 antibody generation}

286 His-tagged TbMCP17 was expressed in E. coli using the pET28a vector (Novagen). The 287 TbMCP17 ORF was PCR amplified using the primers 5'288 ggacggAAGCTTaccatggtttccgagggcacttccgctg-3', $\quad$ 3'- 
289 gcttgcaGGATCCccgttcctccatgaacttcttggc-5' and inserted into the vector using the restriction 290 enzyme sites BamH I and Hind III (underlined). The Lemo21(DE3) E. coli strain was used for 291 His-tagged TbMCP17 expression. Transformed cells were inoculated in LB media with 50 $292 \mu \mathrm{g} / \mathrm{ml}$ kanamycin and $30 \mu \mathrm{g} / \mathrm{ml}$ chloramphenicol and grown overnight. The next day the 293 overnight culture was diluted $1 / 50$ and protein expression was started at $\mathrm{OD}_{600}$ 0.4-0.8 at 37 $294{ }^{\circ} \mathrm{C}$ by the addition of $0.4 \mathrm{mM}$ IPTG for $4 \mathrm{~h}$.

295 Cells were harvested by centrifugation at $4,000 \times \mathrm{g}$ for $10-20 \mathrm{~min}$ at $4{ }^{\circ} \mathrm{C}$, resuspended in 5 $296 \mathrm{ml} / \mathrm{g}$ pellet native resuspension buffer $(50 \mathrm{mM}$ Na-phosphate $\mathrm{pH} 8.0,300 \mathrm{mM} \mathrm{NaCl}$, 297 0.01\%Tween-20) supplemented with protease inhibitor cocktail without EDTA (Sigma), 0.2 $298 \mathrm{mg} / \mathrm{ml}$ lysozyme and 5 unit/ $\mu 1$ DNase. After incubation on ice for 30 min cells were passed 299 through the French press until the lysate turned clear. TbMCP17 inclusion bodies were pelleted 300 at $13,000 \times \mathrm{g}$ for $30 \mathrm{~min}$ and solubilised using $5 \mathrm{M}$ Urea or $1 \%$ sarcosyl. After solubilisation $301 \mathrm{TbMCP} 17$ was allowed to bind to $1 \mathrm{ml}$ of Ni-NTA agarose (Qiagen) $/ 20 \mathrm{ml}$ of solubilised 302 protein, and the mixture was stirred for $60 \mathrm{~min}$ at room temperature. The Ni-NTA agarose was 303 washed according to the manufacturer's protocol and TbMCP17 was eluted using $200 \mu 1$ 304 elution buffer $\left(8 \mathrm{mM} \mathrm{Na}_{2} \mathrm{HPO}_{4}, 286 \mathrm{mM} \mathrm{NaCl}, 1.4 \mathrm{mM} \mathrm{KH}_{2} \mathrm{PO}_{4}, 2.6 \mathrm{mM} \mathrm{KCl}, 500 \mathrm{mM}\right.$ 305 imidazole and $0.1 \%$ sarkosyl (w/v), $\mathrm{pH} 7.4) .3 \mathrm{mg}$ of purified protein were loaded onto a 12 $306 \%$ acrylamide prep-gel and dyed with Coomassie Brilliant Blue R-250. The protein band was 307 cut out and sent to ThermoFisher Scientific for antibody generation. For western blot analysis 308 the antiserum was diluted 1:250 in TBST containing 5\% fish gelatine (Sigma) and detected 309 using an anti-rabbit IgG HRP-conjugated secondary antibody (Abcam) diluted 1:2000 in 310 gelatine/TBST. 


\section{Sequence analyses suggest that $\mathrm{TbMCP17}$ is a mitochondrial iron transporter}

315 Genome database searches using the amino acid sequences of the functionally characterised

316 mitochondrial iron transporters from S. cerevisiae (MRS3 and MRS4) identified only one

317 homologue in the genome of T. brucei, TbMCP17 (accession number Tb927.3.2980; [36]).

318 Reciprocal BLASTP analysis of eukaryotic protein databases (http://www.ncbi.nlm.nih.gov)

319 using the TbMCP17 amino acid sequence retrieved mitochondrial iron transporters from

320 different species, including yeast MRS3 and MRS4, and mitoferrins from plants, mammals

321 and insects. Syntenic homologues are also present in all other kinetoplastids and the free-living

322 Bodo saltans; they include Leishmania major LmjF.29.2780 and Trypanosoma cruzi

323 TcCLB.508153.630, and TcCLB.510315.20.

324 The phylogenetic relationship of TbMCP17 with mitochondrial iron carriers of other 325 eukaryotes was analysed using a neighbour-joining tree (Figure 1). 


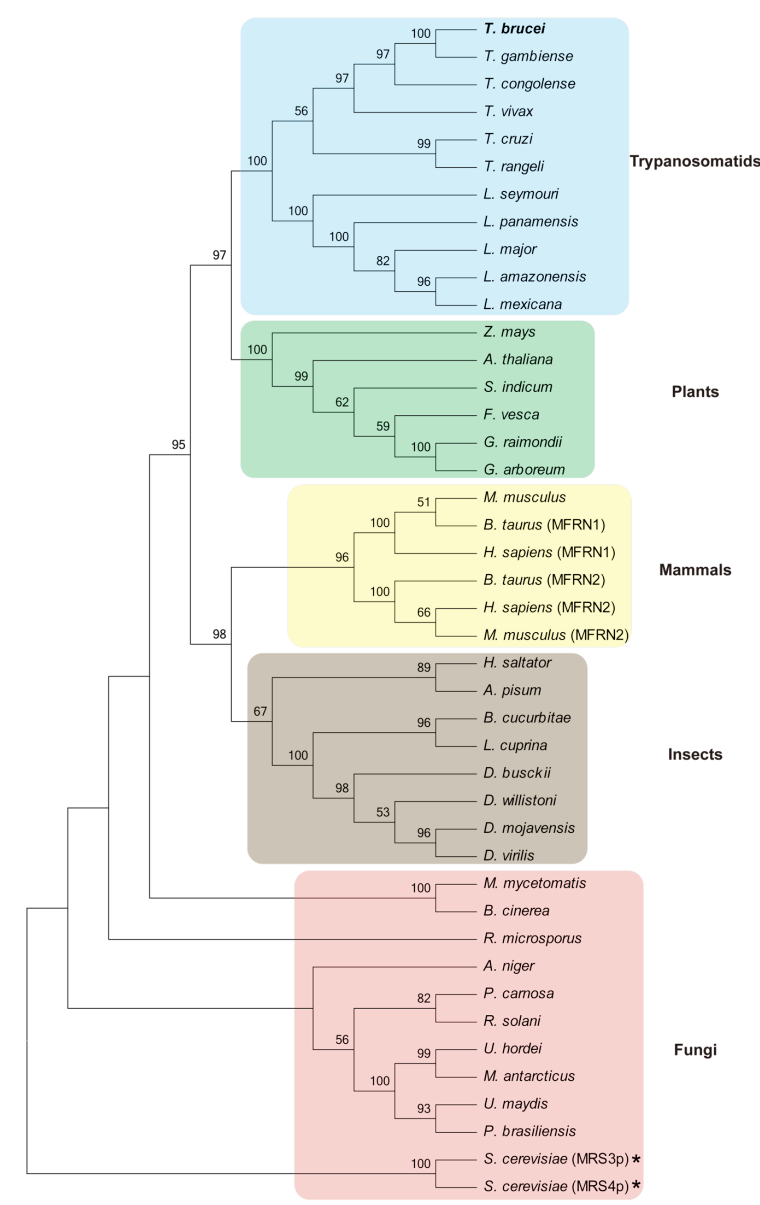

327 Figure 1. TbMCP17 is evolutionarily related to mitochondrial iron transporters of the SLC25A

328 family.

329 The evolutionary relationship was analysed using a Neighbour-Joining tree. The bootstrap

330 consensus tree was obtained after resampling analysis of 1,000 reiterated data sets. Only

331 significant bootstrap values $(\geq 50 \%)$ are shown. Branches corresponding to partitions

332 reproduced in less than 50\% bootstraps are collapsed. TbMCP17 is highlighted by bold face.

333 Functionally characterised mitochondrial iron transporters are labelled with '*'.

334 Corresponding accession numbers are indicated in the materials and methods section.

$336 \mathrm{TbMCP} 17$ and homologous sequences from other trypanosomatids formed a well-defined,

337 separate clade branching off near the iron carriers of plants with a well-resolved node and 338 closely related to the mammalian mitoferrins and the iron carriers of insects. In mammals there 
339 are two different isoforms of mitoferrins, MFRN1 and MFRN2 [31,54], forming two separate

340 branches within the mammalian clade and supported by high bootstrap values (Figure 1). As

341 expected, mitochondrial iron transporters from insects and mammals branched nearer to each

342 other than to the iron carriers from plants and trypanosomatids (Figure 1). Rather surprisingly,

343 instead of branching with the mammalian proteins, those from yeast formed an independent

344 group that was not supported by high bootstrap values (Figure 1).

345 The deduced amino acid sequence of TbMCP17 consists of 289 amino acid residues, which

346 corresponds to a predicted molecular weight of $31.1 \mathrm{kDa}$ (GeneDB). TbMCP17 contains three

347 repetitive domains of about 100 amino acids, each harbouring two membrane-spanning alpha-

348 helices (Figure 2, H1-H6).
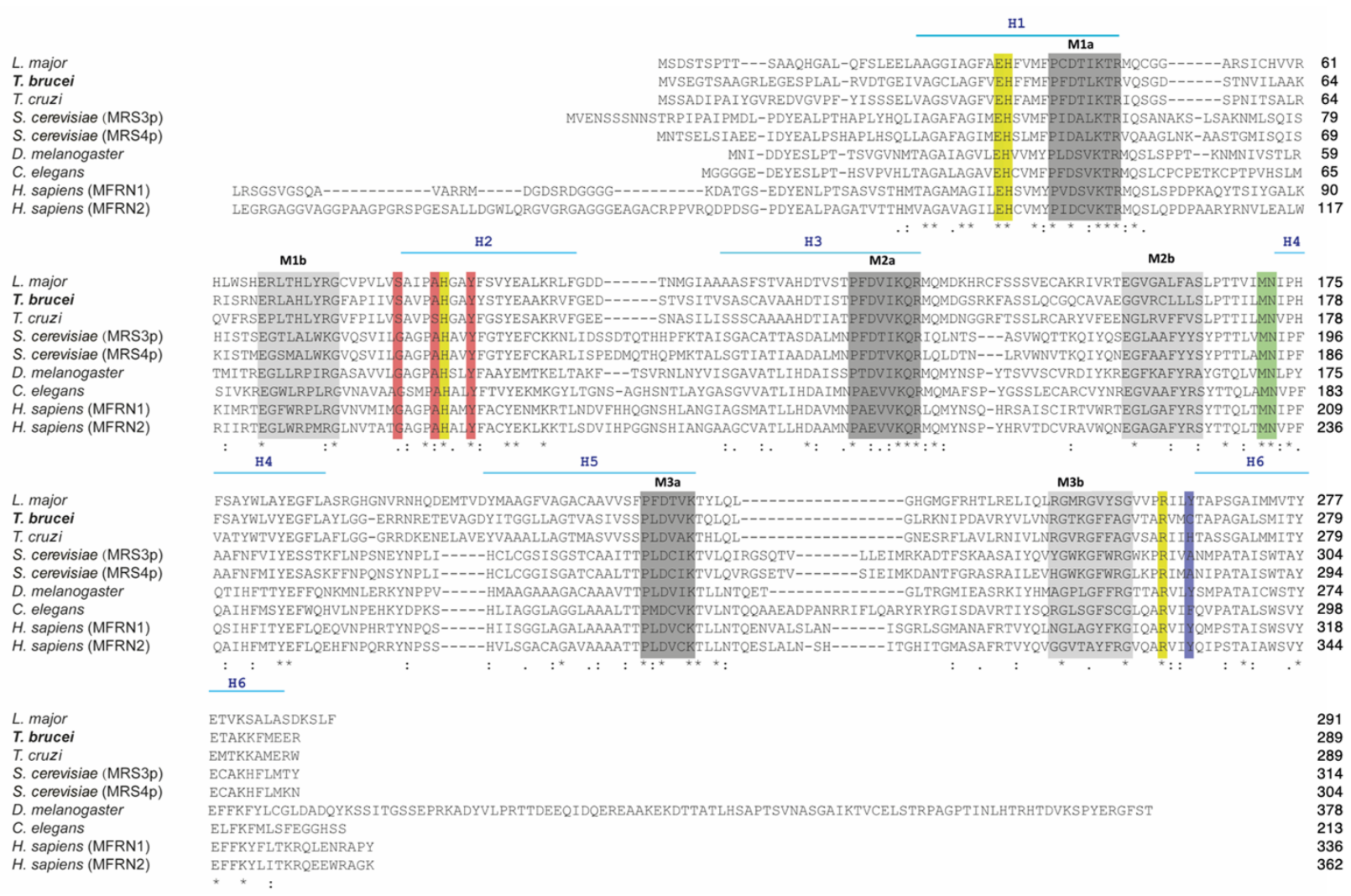

351 Figure 2. TbMCP17 displays all conserved amino acid sequence features and functional

352 residues present in mitochondrial iron carriers. 
353 Sequence alignment of $T b \mathrm{MCP} 17$ with putative mitochondrial iron carrier sequences from

354 Trypanosoma cruzi and Leishmania major, MRS3p and MRS4p Saccharomyces cerevisiae

355 and mitoferrins from Drosophila melanogaster, Caenorhabditis elegans and Homo sapiens.

356 The six transmembrane helices are indicated using a blue line (H1-6). The first and second 357 part of the canonical signature sequence motifs are labelled with M1a, M2a and M3a, and $358 \mathrm{M} 1 \mathrm{~b}, \mathrm{M} 2 \mathrm{~b}$ and M3b and are shaded in dark and light grey respectively. Substrate contact points 359 are shaded in red (CPI), green (CPII) and blue (CPIII). The salt bridge networks are shaded in 360 yellow. (*) indicate fully conserved residues, (:) indicate strongly conserved (scoring $>0.5$ in 361 the Gonnet PAM 250 matrix), (.) indicate weakly conserved (scoring $<0.5$ in the Gonnet PAM 362250 matrix). Corresponding accession numbers are indicated in the materials and methods 363 section.

365 Characteristic for MCF proteins is a bipartite signature motif: the first part of the signature 366 motif $\left(\operatorname{Px}[\mathrm{D} / \mathrm{E}] \mathrm{x}_{2}[\mathrm{~K} / \mathrm{R}] \mathrm{x}[\mathrm{K} / \mathrm{R}]\right.$, $\mathrm{x}$ represents any residue) (Figure 2, M1a, M2a, and M3a) is 367 located at the carboxy-terminal end of the odd-numbered transmembrane helices, and the 368 second part of the signature motif $\left([\mathrm{D} / \mathrm{E}] \mathrm{Gx}_{4-5}[\mathrm{~W} / \mathrm{F} / \mathrm{Y}][\mathrm{K} / \mathrm{R}] \mathrm{G}\right)$ (Figure 2, M1b, M2b, and 369 M3b) shortly before each even-numbered transmembrane helix, within an aliphatic loop [55370 57]. Multiple sequence alignments confirmed the conservation of the MCF-typical signature 371 sequences (M1a/M2a/M3a) in TbMCP17 with some minor modifications (Figure 2). Within 372 the first part of the signature sequence the proline at position one, the aspartic acid at position 3733 and the lysine at position 6 were conserved in TbMCP17 (Figure 2). The positively charged 374 amino acid, either lysine or arginine, at position 8 was also conserved except in M3a (Figure 375 2). The second part of the sequence signature (M1b/M2b/M3b) was less conserved except for 376 the initial acidic residue (D or E) (Figure 2). In M1b the only substitution was at position 2 377 where glycine was substituted for arginine. In M2b the last 2 amino acids of the motif $([\mathrm{K} / \mathrm{R}] \mathrm{G})$ 
378 and the preceding aromatic amino acid $[\mathrm{W} / \mathrm{F} / \mathrm{Y}]$ were not conserved in $T b \mathrm{MCP} 17$. M3b was

379 little conserved and retained only the final glycine (Figure 2). The other aligned sequences 380 displayed similar levels of conservation in their signature motifs.

381 In MCF proteins the substrate specificity is determined by three well-conserved substrate382 contact points (CPI, CPII and CPIII) (marked respectively red, green and blue in Figure 2) 383 [35]. In TbMCP17, like in all iron carriers, the amino acids constituting CPI and CPII are $384[\mathrm{G} / \mathrm{S}] \mathrm{AY}$ and MN respectively while CPIII is not conserved (Figure 2). Three further repetitive 385 clusters of conserved residues ([FY][DE]XX[RK]) located in H2, H4 and H6 (marked yellow 386 in Figure 2) are thought to be involved in the formation of salt bridge networks [58]. Except 387 for the last amino acid in the motif located in $\mathrm{H} 4$ the other repetitive clusters are well conserved 388 in all aligned sequences (Figure 2).

\section{TbMCP17 complements growth in $\triangle \mathrm{MRS3} / 4$ yeast}

391 To assess the function of $T b \mathrm{MCP} 17$ as potential mitochondrial iron importer, functional 392 complementation was performed in $\Delta \mathrm{MRS} 3 / 4$ S. cerevisiae strains (GW403). The wild type 393 and GW403 yeast strains were transfected using either empty pCM190 or pCM190 containing 394 TbMCP17 or MRS3 and spotted on SCD (glucose) or SCG (glycerol) agar plates. The results 395 showed that GW403 yeast transfected with empty pCM190 grew slower than wild type yeast 396 or GW403 yeast complemented with either TbMCP17 or MRS3 (Figure 3A). This effect was 397 particularly evident when the yeast was grown on glycerol instead of glucose as carbon source 398 (Figure 3A). 
A

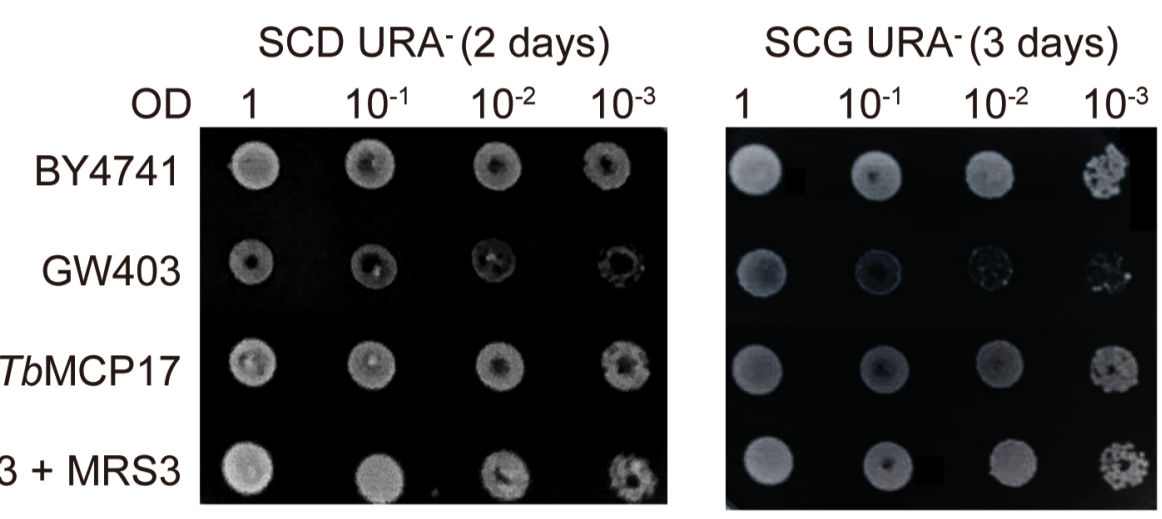

GW403 + MRS3

Fermentable (glucose) Non fermentable (glycerol)
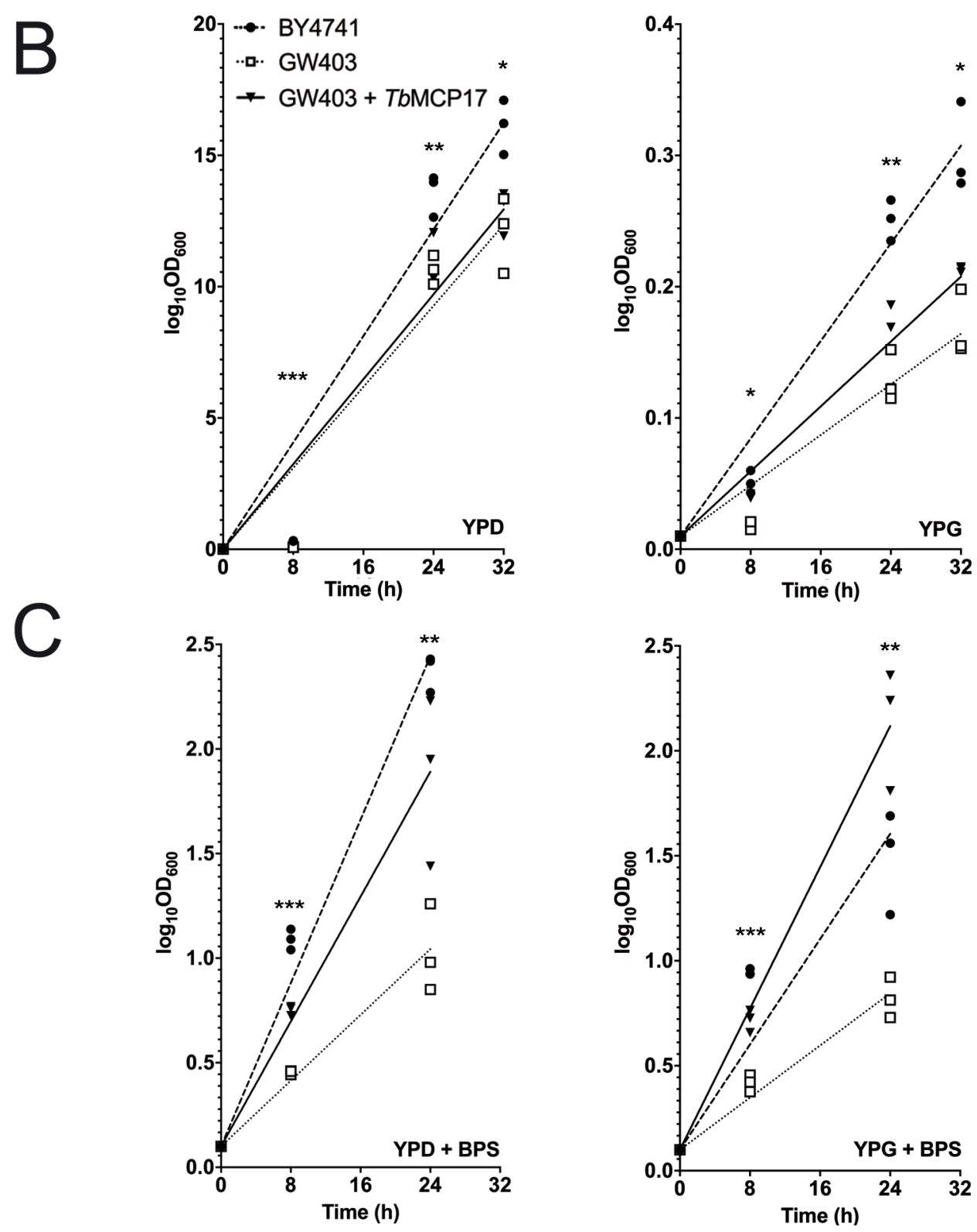

400

401 Figure 3. TbMCP17 complements the growth defect of the $\Delta \mathrm{MRS} 3 / 4$ yeast cell line. 
402 A. The yeast strain BY4741 (wild-type) was transfected with empty pCM190 vector and the 403 yeast strain GW403 ( $\triangle \mathrm{MRS} 3 / 4)$ was transfected with either empty pCM190 vector or with 404 pCM190 vector containing either the TbMCP17 or the MRS3 ORF. Transfected yeast cells 405 were spotted in ten-fold dilutions on plates containing synthetic complete media without uracil 406 supplemented with either dextrose (SCD URA ${ }^{-}$) or glycerol (SCG URA $\left.{ }^{-}\right)$. B. and $\mathbf{C}$. The yeast 407 strain BY4741 (wild-type) was transfected with empty pCM190 vector and the yeast strain $408 \Delta \mathrm{MRS} 3 / 4$ was transfected with either empty pCM190 vector or with pCM190 vector 409 containing the TbMCP17 ORF. Transfected cells were grown either in standard YPD or YPG 410 (B) or YPD or YPG supplemented with $80 \mu \mathrm{M}$ bathophenanthrolinedisulfonic acid (BPS) (C).

411 For each yeast strain three individual growth curves were plotted. Statistical significance was 412 determined by one-way ANOVA using GraphPad Prism 7: *: $p \leq 0.05 * *: p \leq 0.01 ; * * * \leq \leq$ 4130.001.

415 Next, the growth rates of the different yeast strains were analysed in YPD (glucose), YPG 416 (glycerol), YPD/BPS (glucose and iron chelator) and YPG/BPS (glycerol and iron chelator) 417 suspension culture media over a period of $32 \mathrm{~h}$ or $24 \mathrm{~h}$ (for experiments with BPS). The best 418 growth rate for all tested yeast cell lines was observed on YPD medium. In this medium no 419 difference in growth was observed between $\triangle \mathrm{MRS} 3 / 4$ cells transfected with either $T b \mathrm{MCP} 17$ 420 or empty vector, which all grew slightly slower than the wild type strain (Figure 3B). In YPG 421 media, the $\Delta \mathrm{MRS} 3 / 4$ and the $\Delta \mathrm{MRS} 3 / 4$ cell line transfected with empty vector grew 422 significantly slower than wild type cells (Figure 3B). Transfection of the $\Delta \mathrm{MRS} 3 / 4$ line with 423 TbMCP17 partially rescued the growth to normal levels compared to the strain transfected 424 with empty vector (Figure 3B). The addition of the iron chelator BPS resulted in drastically 425 reduced growth rates for all strains on YPD and YPG medium compared with the growth 426 without iron chelator (Figure 3C). However, in both media containing BPS, TbMCP17 
427 improved the growth of the $\Delta \mathrm{MRS} 3 / 4$ strain relatively to the strain transfected with empty

428 vector (Figure 3C). Taken together these results demonstrated that $\mathrm{TbMCP} 17$ partially restores 429 growth of the $\Delta \mathrm{MRS} 3 / 4$ strain by complementing the transport function of the yeast iron 430 carriers.

\section{TbMCP17 localises to the mitochondrion of PCF T. brucei}

433 Results from one of our previous publications demonstrated that the iron carrier, together with 434 the dicarboxylate carrier TbMCP12, are the only T. brucei MCF carriers to be differentially 435 expressed at the mRNA level [36,39]. Since those results showed that TbMCP17 is 436 approximately 2-fold higher expressed in PCF than in BSF [36] and because we did not obtain 437 viable BSF clones over expressing TbMCP17, in the current work we focussed on its 438 functional characterisation in PCF only.

439 Several attempts to detect endogenous TbMCP17 in PCF using the antibody we generated 440 were not successful, although the antibody readily detected TbMCP17 in PCF when the protein 441 was overexpressed (Supplementary Figure S1). When using our standard culturing conditions $442 T b \mathrm{MCP} 17$ was therefore below the detection limit of the antibody.

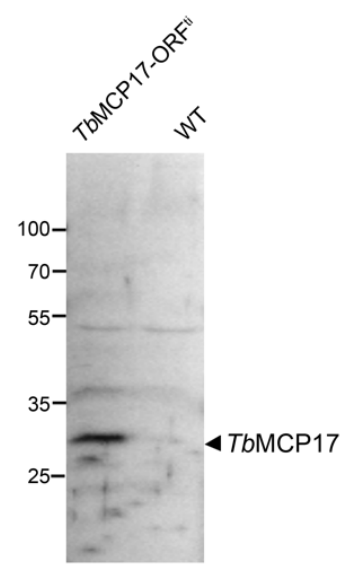


444 Supplementary Figure S1. Western blot analysis of TbMCP17 using the antibody we 445 generated in wild type PCF and PCF overexpressing TbMCP17. In each lane $10 \mu 1$ of sample 446 containing $5 \times 10^{6}$ cells were loaded.

448 Since the location of mitochondrial targeting sequences in proteins belonging to the SLC25A 449 protein family has not been fully clarified yet [59-61] we assessed the subcellular localisation 450 of TbMCP17 by over-expressing it bearing either a C-terminal or N-terminal myc-tag in PCF 451 (MCP17-cmyc ${ }^{\mathrm{ti}}$ and MCP17-nmyc $\mathrm{c}^{\mathrm{ti}}$ ). In this way we ensure that the myc-tag is not interfering 452 with the mitochondrial localisation of the protein. Following transfection of PCF T. brucei 453 with the over expression constructs, western blot analysis using an antibody directed against 454 the myc-tag showed a band of approximately $33 \mathrm{kDa}$ for both the TbMCP17-cmyc ${ }^{\mathrm{ti}}$ and the 455 TbMCP17-nmyc ${ }^{\text {ti }}$ PCF cell lines, but not for the wild type cells (Figure 4A). Leakiness of the 456 tet-inducible expression-system in the absence of tetracycline $[39,48]$ was not detectable by 457 western blotting but cannot be excluded. Therefore wild-type cells were used as control cell458 line in all experiments. Immunofluorescence analysis using Mitotracker and an antibody 459 directed against the myc-tag showed that both $\mathrm{N}$ - and C-terminally tagged TbMCP17 are 460 localised to the mitochondrion (Figure 4B). 
A
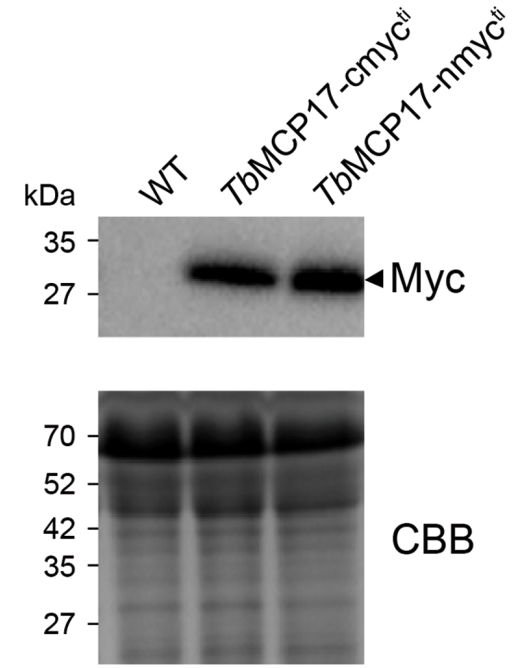

CBB

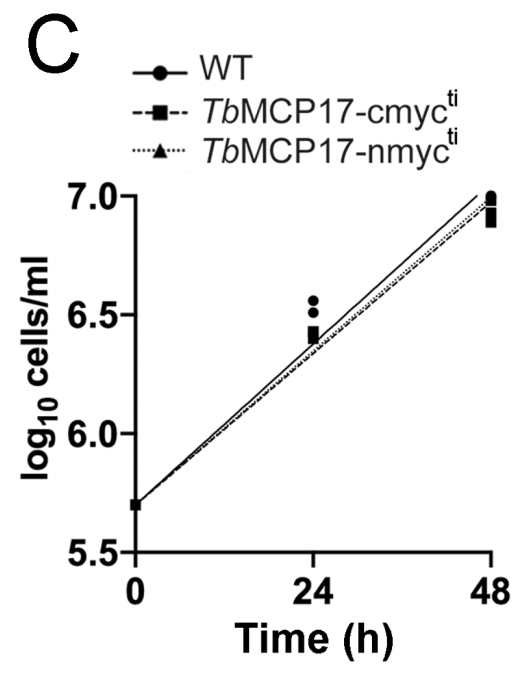

$0 \mu \mathrm{M}$ deferoxamine
B
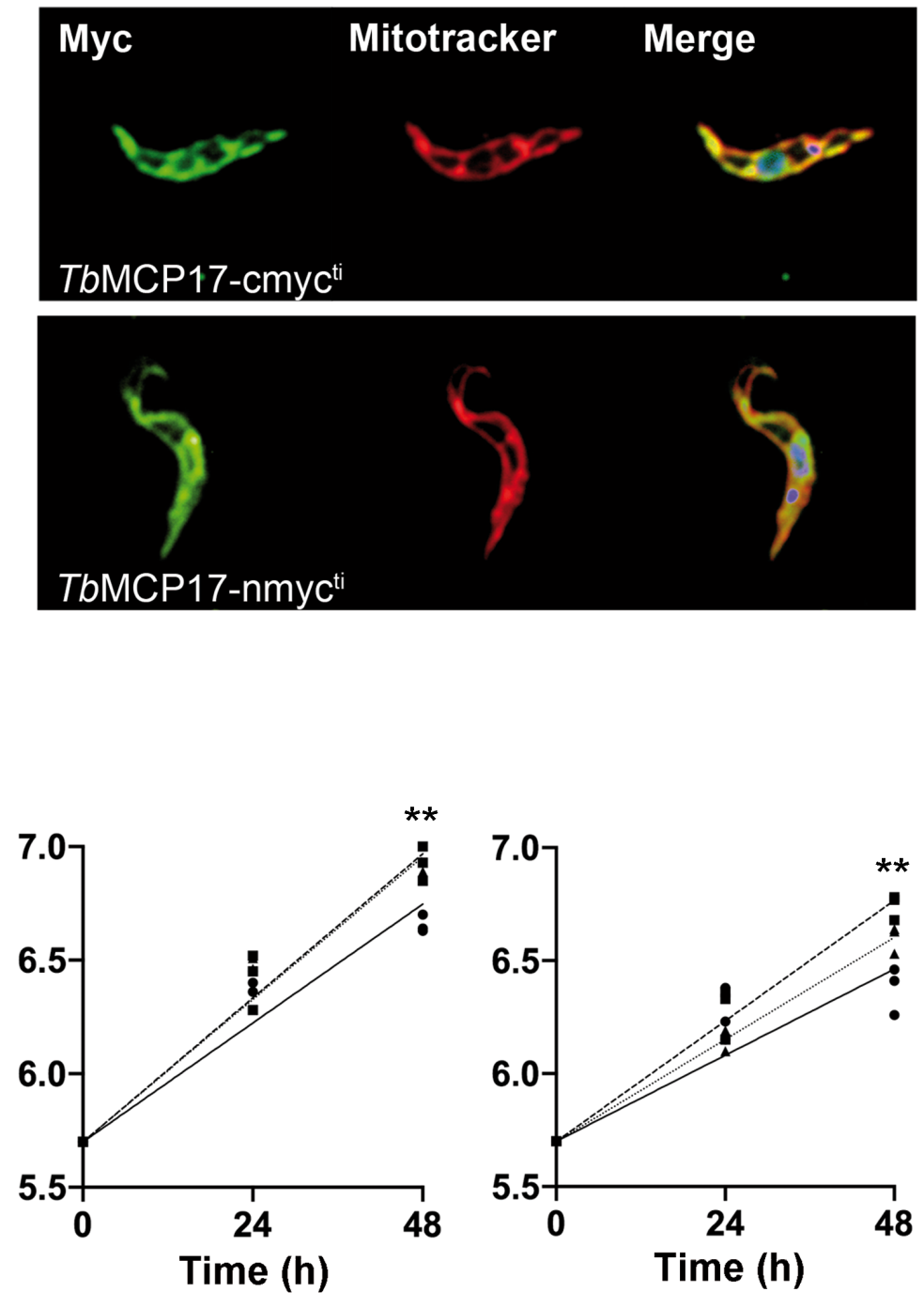

$50 \mu \mathrm{M}$ deferoxamine

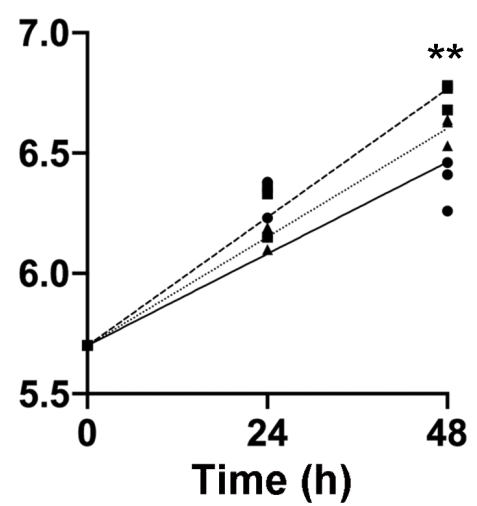

$100 \mu \mathrm{M}$ deferoxamine

461

462 Figure 4. In PCF TbMCP17 is localised in the mitochondrion and its overexpression confers 463 increased resistance to iron depletion.

464 A. Western blot analysis of WT PCF and PCF over-expressing TbMCP17 with either C-term 465 (TbMCP17-cmyc $\left.c^{\mathrm{ti}}\right)$ or N-term myc-tag (TbMCP17-nmyc $\left.{ }^{\mathrm{ti}}\right)$. Coomassie brilliant blue (CBB) is 466 used as loading control. B. Immunofluorescence analysis of PCF over expressing TbMCP17 467 bearing either C-term (TbMCP17-cmyc $\left.c^{\mathrm{ti}}\right)$ or N-term myc-tag (TbMCP17-nmyc $\left.{ }^{\mathrm{ti}}\right)$. 468 Mitochondria were visualised using Mitotracker (red) and TbMCP17-myc was detected using 
469 a 1:1000 dilution of a myc antibody (green). Nucleus and kinetoplasts are stained with DAPI

470 (blue). C. Growth analysis of PCF wild-type (WT), TbMCP17-cmyc ${ }^{\mathrm{ti}}$ and TbMCP17-nmyc ${ }^{\mathrm{ti}}$

471 cells in the absence and presence of the iron chelator deferoxamine. The growth experiment

472 was started at a cell density of $0.5 \times 10^{6}$ cells $/ \mathrm{ml}$ and cells were counted every $24 \mathrm{~h}$ for a period

473 of $48 \mathrm{~h}$. In some experiments, cells were treated with either 50 or $100 \mu \mathrm{M}$ deferoxamine. For

474 each cell-line three individual growth curves were plotted. Statistical significance was

475 determined by one-way ANOVA using GraphPad Prism 7: *: $p \leq 0.05 * *: p \leq 0.01 ; * * * p \leq$ 4760.001.

477

478 Over expression of $\mathbf{T b}$ MCP17 sustains growth under iron-limiting condition in PCF $\boldsymbol{T}$.

479 brucei

480 To investigate whether a surplus of TbMCP17 enabled better survival of T. brucei under iron481 limiting conditions $T b \mathrm{MCP} 17-\mathrm{cmyc}^{\mathrm{ti}}$ and $T b \mathrm{MCP} 17-\mathrm{nmyc}^{\mathrm{ti}}$ procyclic cell lines were 482 cultivated in medium containing different concentrations of the iron chelator deferoxamine. 483 Deferoxamine is a conventionally used and well-established compound for blocking iron 484 uptake in trypanosomes [62]. In contrast to BPS, it is membrane-permeable and chelates 485 intracellular iron thereby blocking its incorporation into newly synthesized proteins [62]. In 486 medium containing no deferoxamine, TbMCP17-cmyc ${ }^{\mathrm{ti}}$ and $T b \mathrm{MCP} 17-\mathrm{nmyc} \mathrm{c}^{\mathrm{ti}}$ cells doubled 487 at the same speed as wild type cells (Figure 4C). The division time of wild type cells when 488 exposed to $50 \mu \mathrm{M}$ or $100 \mu \mathrm{M}$ deferoxamine for $48 \mathrm{~h}$ was significantly slowed down compared 489 to the cells overexpressing $T b \mathrm{MCP} 17$ (respectively $p=0.0012$ and $p=0.0031$ ) or untreated 490 cells (respectively $p=0.0012$ and $p=0.0042$ ) (Figure 4C). Instead, the division time of 491 TbMCP17-cmyc ${ }^{\mathrm{ti}}$ and TbMCP17-nmyc ${ }^{\mathrm{ti}}$ was not affected by the exposure to $50 \mu \mathrm{M}$ iron 492 chelator and only slightly but not significantly slowed down when exposed to $100 \mu \mathrm{M}$ iron 
493 chelator (Figure 4C). These results indicate that the overexpression of TbMCP17 confers

494 increased resistance to iron depletion.

495

496 TbMCP17 is required to sustain normal growth of PCF T. brucei

497 We were next interested to investigate whether TbMCP17 was essential for procyclic $T$. brucei 498 viability. To deplete TbMCP17 we used two different approaches: conditional gene knock out 499 and RNA interference (RNAi).

500 For the conditional gene knock out we replaced the two alleles of TbMCP17 with antibiotic 501 resistant cassettes in the background of the tetracycline inducible $T b \mathrm{MCP} 17-\mathrm{cmyc}^{\mathrm{ti}}$ generating 502 the cell-line $\triangle T b \mathrm{MCP} 17 / T b \mathrm{MCP} 17-\mathrm{cmyc}^{\mathrm{ti}}$ according to the previously published method [37503 39]. PCR using different primer sets located along the modified TbMCP17 gene locus 504 (Supplementary Table 1) was performed to confirm the removal of the TbMCP17 gene and 505 the correct insertion of the resistance cassettes (Supplementary Figure 2).

506

\begin{tabular}{|c|l|l|}
\hline Primer number & \multicolumn{1}{|c|}{ Primer name } & \multicolumn{1}{c|}{ Primer sequence } \\
\hline 427 & MCP17upstreamFor & gatgatcgtatcggctcttgtcgcaatg \\
\hline 339 & MCP17KO5ForSac & gctagagctccgtgtcgtgaggtggagaggtgatg \\
\hline 429 & NEOFor & atgcggcggctggatacggttg \\
\hline 82 & BSDFor & atggccaagcctttgtctcaagaagaatccac \\
\hline 83 & BSDRev & ttagccctcccacacataaccagagg \\
\hline 105 & NeoRev & tcagaagaactcgtcaagaaggcgatagaag \\
\hline 430 & NeoRev2 & cgagccctgatgctcttcgtccagatcatcctgatc \\
\hline 431 & MCP17downstreamRev & aaggagtgggaacaggggcaaatccac \\
\hline 338 & MCP17KO3RevA & ctatgggcccgtcaaacacattactggagcgg \\
\hline
\end{tabular}

507 Supplementary Table 1. Primers used for checking the correct integration of the knock out 508 construct. 


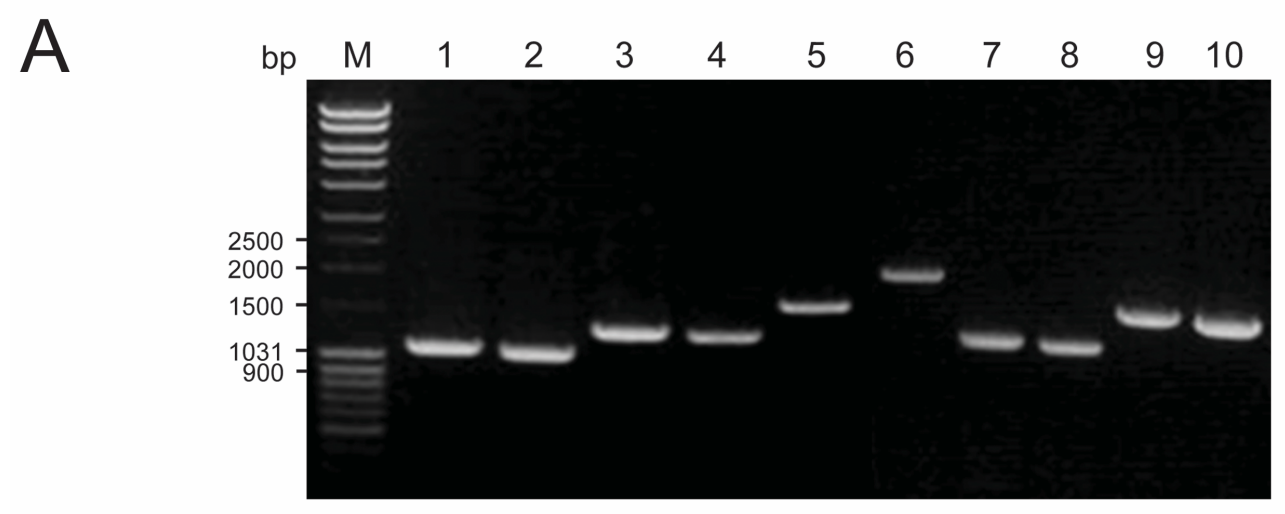

B

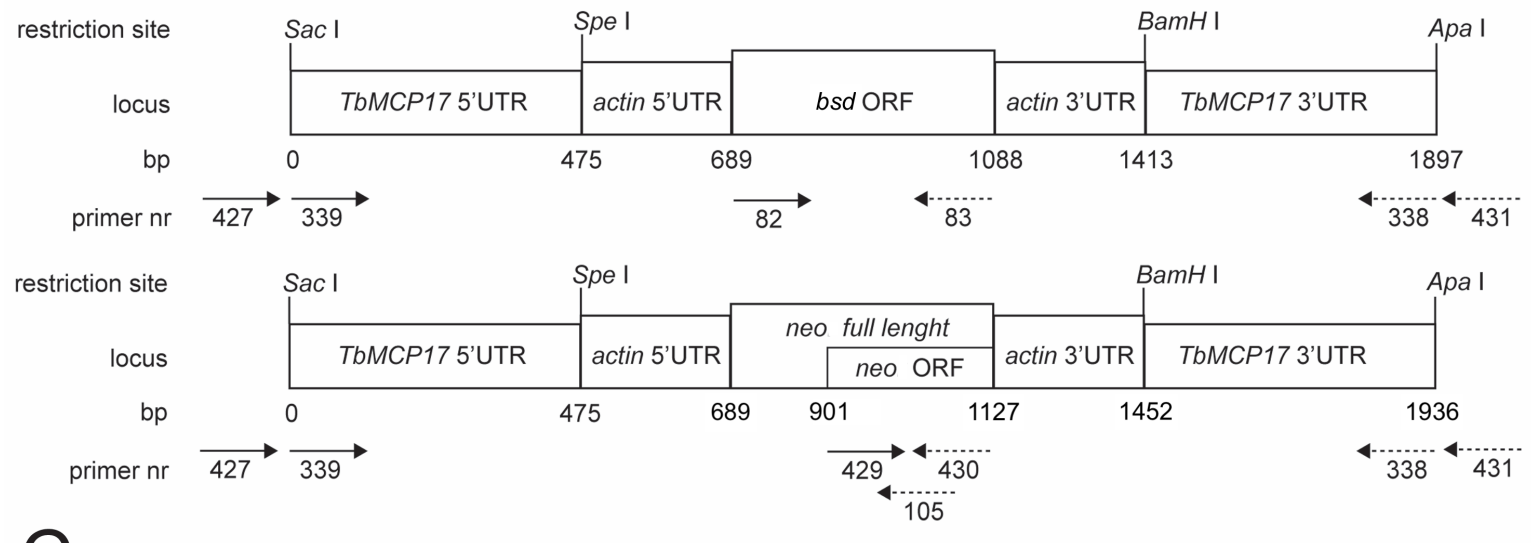

C

\begin{tabular}{|c|c|c|c|}
\hline $\begin{array}{c}\text { PCR } \\
\text { number }\end{array}$ & Forward primer & Reverse primer & $\begin{array}{c}\text { Predicted } \\
\text { product size } \\
(\mathrm{bp})\end{array}$ \\
\hline 1 & 427 & 83 & 1116 \\
\hline 2 & 339 & 83 & 1088 \\
\hline 3 & 427 & 105 & 1079 \\
\hline 4 & 339 & 105 & 1051 \\
\hline 5 & 427 & 430 & 1155 \\
\hline 6 & 339 & 431 & 1964 \\
\hline 7 & 429 & 431 & 1062 \\
\hline 8 & 429 & 338 & 1035 \\
\hline 9 & 82 & 431 & 1235 \\
\hline 10 & 82 & 338 & 1208 \\
\hline
\end{tabular}

511 Supplementary Figure 2. PCR analysis of the correct integration of the antibiotic resistance

512 cassette in the $\triangle T b \mathrm{MCP} 17 / T b \mathrm{MCP} 17-\mathrm{cmyc}^{\mathrm{ti}}$ cell-line. A. gDNA derived from

$513 \Delta T b \mathrm{MCP} 17 / T b \mathrm{MCP} 17-\mathrm{cmyc}^{\mathrm{ti}}$ was PCR amplified and loaded on a $0.5 \%$ agarose gel together

514 with a DNA ladder. B. Schematic representation of the location of the primers used in A. 
515 within the recombination site at the TbMCP17 gene locus. C. List of the primers used in A. 516 and their predicted PCR amplified DNA fragment size.

518 To check the correct integration of the antibiotic resistance cassettes, primers located upstream 519 (427) and downstream (431) of the homologous recombination site were used in combination 520 with primers located in the BSD (82 and 83) or NEO $(105,429,430)$ ORFs (Supplementary 521 Figure 2 C). All PCRs performed using genomic DNA derived from the TbMCP17 knock out 522 cells produced a fragment of the predicted length confirming the integration of the resistance 523 genes in the TbMCP17 locus (Supplementary Figure $2 \mathrm{~A}$ and C). Tetracycline removal from 524 the culture depletes the myc-tagged TbMCP17 (Figure 5A). This cell line was referred to as 525 TbMCP17-cmyc ${ }^{\mathrm{ti}}$ depleted knock out (TbMCP17 KO). 
A

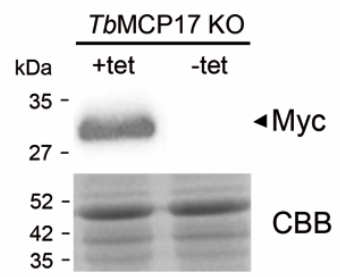

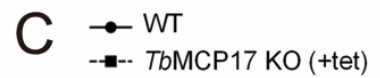

-... TbMCP17 KO (-tet)

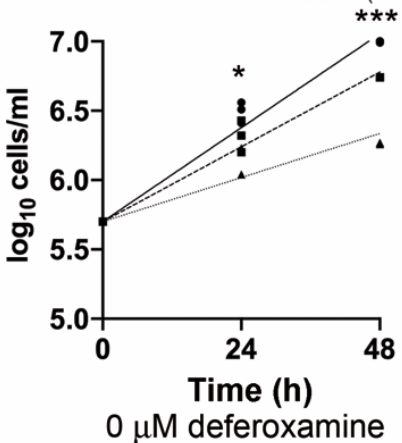

D $\rightarrow W T$

-- TbMCP17 RNAi

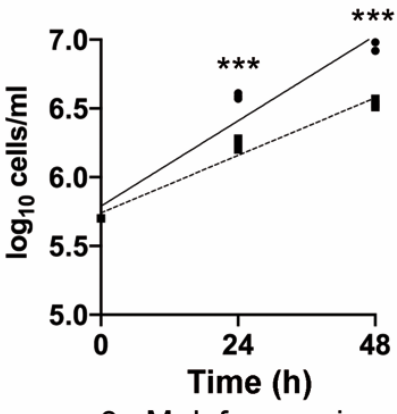

$0 \mu \mathrm{M}$ deferoxamine
B

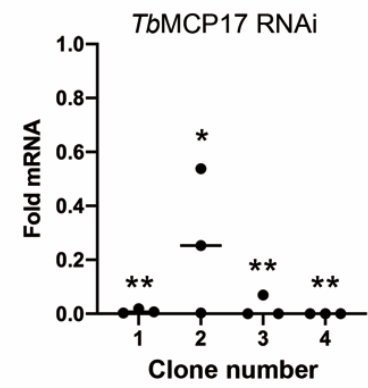

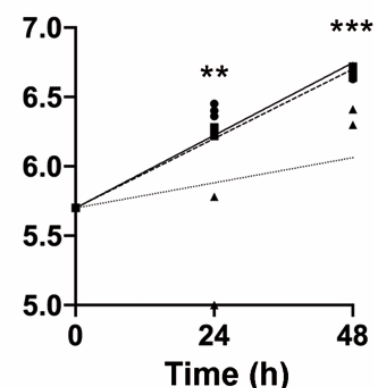

$50 \mu \mathrm{M}$ deferoxamine

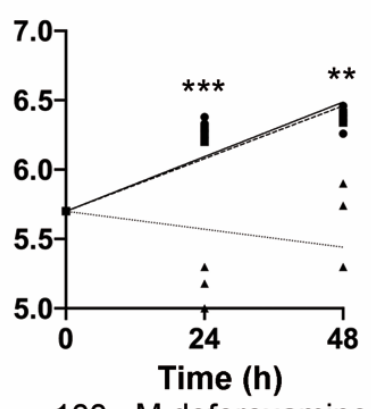

$100 \mu \mathrm{M}$ deferoxamine

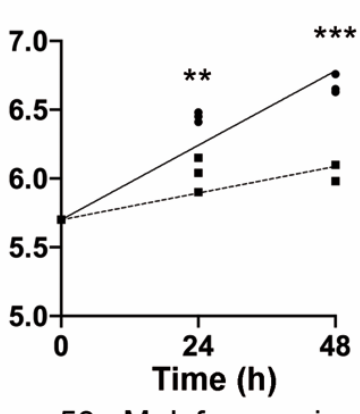

$50 \mu \mathrm{M}$ deferoxamine

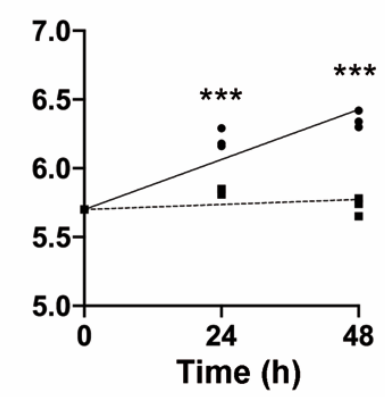

$100 \mu \mathrm{M}$ deferoxamine

527 Figure 5. TbMCP17 depletion impairs PCF T. brucei growth and renders the cells more

528 susceptible to iron depletion.

529 A. Western blot analysis of TbMCP17 KO grown in the presence and absence of tetracycline

530 for $48 \mathrm{~h}$. Coomassie brilliant blue (CBB) is used as loading control. B. qPCR analysis of the

531 TbMCP17 expression of 4 different $T b \mathrm{MCP} 17 \mathrm{RNAi}$ clones harvested after $48 \mathrm{~h}$ of growth in

532 the presence of tetracycline. TbMCP17 expression of WT PCF was set to 1. Graph represents

533 the mean of 3 independent experimental replicates and individual values are plotted. Statistical

534 significance was determined by one-way ANOVA using GraphPad Prism 7: *: $p \leq 0.05 * *: p$

$535 \leq 0.01 ; * * * p \leq 0.001$. C. and D. Growth analysis of PCF wild-type (WT) and TbMCP17 KO 
536 (+ and - tet) PCF cells $(\mathbf{C})$ or TbMCP17 RNAi PCF cells $(\mathbf{D})$ in the absence or presence of the

537 iron chelator deferoxamine. The analysis of the growth curve was started at a cell density of

$5380.5 \times 10^{6}$ cells $/ \mathrm{ml}$ and cells were counted every $24 \mathrm{~h}$ for a period of $48 \mathrm{~h}$. In some experiments,

539 cells were treated with either 50 or $100 \mu \mathrm{M}$ deferoxamine. For each cell-line three individual

540 growth curves were plotted. Statistical significance was determined by one-way ANOVA

541 using GraphPad Prism 7: *: $p \leq 0.05 * *: p \leq 0.01 ; * * * p \leq 0.001$.

543 The efficacy of 4 TbMCP17 RNAi clones was tested using RT-qPCR. Compared to wildtype 544 cells, in three clones we found over 100-fold reduction of the mRNA expression of $T b \mathrm{MCP} 17$

545 (clones 1, 3 and 4), while in one (clone 2) only 4-fold reduction was achieved (Figure 5B).

546 In medium containing no deferoxamine, the doubling time of the TbMCP17-cmyc ${ }^{\text {ti }}$ depleted

$547 \mathrm{KO}$ was significantly slowed down compared to either wild type or induced TbMCP17 KO

548 (Figure 5C). A similar increase in doubling time compared to wild type cells was obtained

549 when $T b \mathrm{MCP} 17$ was depleted by RNAi when no iron chelator was added to the culture (Figure $5505 \mathrm{D})$.

551 The doubling time in iron-depleting conditions ( 50 or $100 \mu \mathrm{M}$ deferoxamine) was significantly 552 slowed down in the TbMCP17-cmycti depleted KO cells compared to wild type cells and 553 induced TbMCP17 KO cells (Figure 5C). The TbMCP17-cmycti depleted KO, however, 554 displayed a slight recovery after $48 \mathrm{~h}$ in culture. Like the $T b \mathrm{MCP} 17-$ cmyc $^{\text {ti }}$ depleted $\mathrm{KO}$, also 555 the TbMCP17 RNAi cells displayed significantly increased sensitivity to iron depletion 556 (Figure 5D)

\section{Mitochondrial iron content is decreased in $\mathrm{TbMCP17}$ depleted cells}

559 We next measured the iron content of total cell lysates and isolated mitochondria in wild type, 560 TbMCP17 $\mathrm{cmyc}^{\mathrm{ti}}$ and TbMCP17 RNAi cell lines. In standard medium, wild type and 
$561 T b \mathrm{MCP} 17 \mathrm{cmyc}^{\mathrm{ti}}$ cells presented similar iron content in both whole cell lysates and

562 mitochondria as well as comparable iron distribution percentage $\left(\mathrm{Fe}_{\mathrm{mito}}: \mathrm{Fe}_{\text {total }}\right)$ (Figure 6A and

563 C). TbMCP17 RNAi cell lines instead presented a slight, though not significant increment of 564 the whole cell iron content compared to wild type and TbMCP17cmycti cells, while the 565 mitochondrial iron content was unchanged.
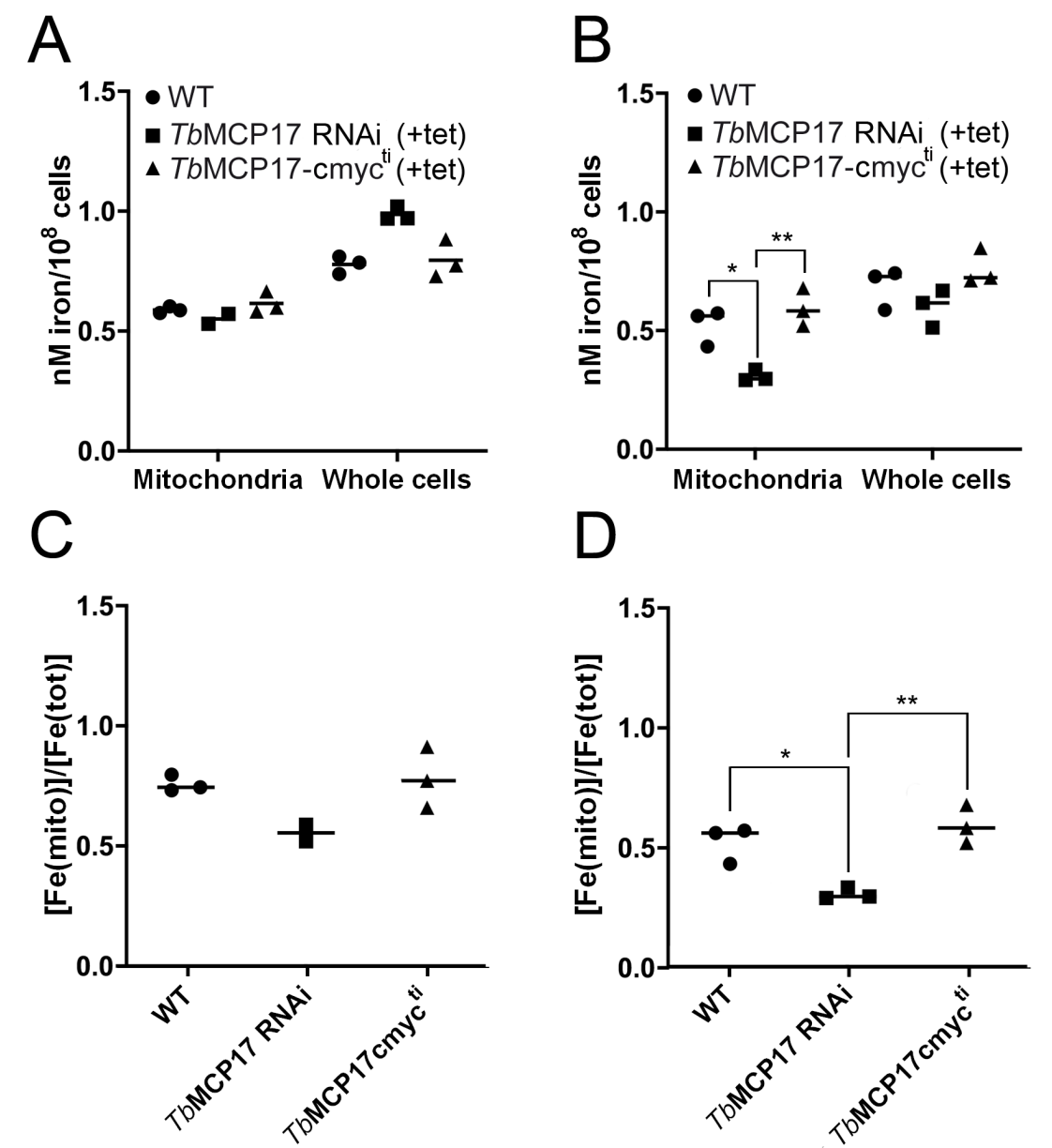

568 Figure 6. Mitochondrial iron content is reduced by the depletion of TbMCP17 only under iron 569 limiting conditions.

570 A. and B. The iron content was measured in whole cell extracts and isolated mitochondria 571 derived from $1 \times 10^{8} \mathrm{WT}, T b \mathrm{MCP} 17 \mathrm{cmyc}^{\mathrm{ti}}$ and TbMCP17 RNAi PCF cells, which were 572 cultivated in normal (A) or heme-depleted medium (B). C. and D. Iron distribution 
$573\left(\mathrm{Fe}_{\text {mito }}: \mathrm{Fe}_{\text {total }}\right)$ in WT, TbMCP17cmyc ${ }^{\mathrm{ti}}$ and TbMCP17 RNAi PCF cells, which were cultivated

574 in normal (C) or heme-depleted medium (D) medium. All graphs (A, B, C and D) were derived

575 from 3, separately plotted, experiments. Statistical significance was determined by one-way

576 ANOVA using GraphPad Prism 7: *: $\mathrm{p} \leq 0.05 * *: \mathrm{p} \leq 0.01 ; * * \mathrm{p} \leq 0.001$.

577

578 Trypanosomes are auxotrophic for heme $[63,64]$. After heme removal the iron content was

579 significantly decreased only in isolated mitochondria of cells in which TbMCP17 was depleted

580 (Figure 6B). The TbMCP17 RNAi cell-line also displayed a significantly lower iron

581 distribution percentage compared with wild type cells and cells overexpressing $582 T$ TbMCP17 $\mathrm{cmyc}^{\mathrm{ti}}$ (Figure 6D). 
585 The aim of this study was the identification of the carrier that is involved in the transport of 586 iron across the inner mitochondrial membrane of the parasite T. brucei. Our results show that 587 the T. brucei gene TbMCP17, which codes for a member of the SLC25A mitochondrial carrier 588 family is the only homologue of mitochondrial iron transporters present in the genome of this 589 parasite. Phylogenetic and amino acid sequence analysis demonstrated that TbMCP17 is 590 closely related to the iron transporters of plants, insects and mammals and displays all 591 signature motifs and residues typically found in mitochondrial iron carriers. It is interesting 592 that as we previously reported for the mitochondrial dicarboxylate carrier TbMCP12 [39] also 593 TbMCP17 appears to have a closer phylogenetic relationship to plant transporters than to those 594 of Opisthokonts, although Excavata probably diverged equally early from both. Also, several 595 T. brucei enzymes such as fructose-1,6-bisphosphate aldolase and arginine kinases were 596 proposed to have arisen through horizontal gene transfer from either cyanobacteria, algae, 597 plants or insects [39,65-68].

598 TbMCP17 successfully rescued the growth defect of the $\Delta$ MRS3/4 (GW403) yeast strain on 599 non-fermentable carbon sources. This observation strongly suggested that TbMCP17 can 600 partially complement the function of yeast MRS3 and MRS4 on non-fermentable carbon 601 sources by translocating iron into the mitochondrial matrix. Yeast growth was, however, never 602 fully restored to wild type levels; perhaps amino acid sequence variations present in TbMCP17 603 impair either its correct integration into the yeast mitochondrial inner membrane or the kinetics 604 of the transport activity. Similar observations were already reported for the carrier TbMCP12 605 [39].

606 In procyclic $T$. brucei, growth under iron limiting conditions was significantly improved when 607 TbMCP17 was over-expressed, while down-regulation and knock out of TbMCP17 
608 significantly reduced cell viability and caused hypersensitivity to iron deprivation. Similar

609 susceptibility was previously observed when the mucolipin-like protein (TbMLP), an 610 endosomal channel required for the assimilation of iron, was depleted from BSF T. brucei [14].

611 After $48 \mathrm{~h}$ of growth on deferoxamine we observed a partial recovery of the growth phenotype 612 in the $T b \mathrm{MCP} 17-\mathrm{cmyc}^{\mathrm{ti}}$ depleted KO. Reasons for this observation might be: i) metabolic 613 adjustments that compensate the decreased iron availability, ii) the upregulation of the 614 expression of other mitochondrial carriers involved in iron transport eg. TbMCP23 or 615 sideroflexins ( $T b S F N X)$. TbMCP23 is highly homologous to Rim2 [36], which mediates the 616 translocation of iron and other divalent metal ions across the mitochondrial inner membrane.

617 In yeast, the combined removal of Mrs3, Mrs4, and Rim2 caused a more severe Fe-S protein 618 maturation defect than the depletion of the Mrs proteins alone [45]. Sideroflexins were 619 identified in T. brucei and display high sequence similarity to the tricarboxylate transporter 620 SLC25A1 (C. Colasante, unpublished data). The depletion of these proteins causes iron 621 accumulation in mitochondria and siderocytic anaemia in mice [69,70], indicating their 622 potential role in iron transport, iii) Selective pressure caused by the low iron concentration 623 might exacerbate the leakiness of the tetracycline-inducible system generating sufficiently 624 high amounts of $T b \mathrm{MCP} 17$ to improve growth though remaining under the detection limit of 625 the western blot analysis.

626 Our results suggested that iron storage is disturbed when TbMCP17 is absent and enhanced 627 when its abundance is increased. When we analysed the iron content of total cell lysates of 628 procyclic $T$. brucei we found, however, that the mitochondrial iron content and the $629 \mathrm{Fe}($ mito)/Fe(total) ratio were significantly lowered in TbMCP17 RNAi cells under low iron 630 conditions but never significantly elevated when TbMCP17 was overexpressed. This indicated 631 that TbMCP17 downregulation decreased, as expected, mitochondrial iron import, which 632 further substantiates its function as iron carrier. That TbMCP17 overexpression does not 
633 increase mitochondrial iron storage in either normal or iron depleted conditions might be due

634 to regulatory mechanisms that limit mitochondrial iron import. It was indeed previously shown

635 that over expression of Mfrn1 and Mfrn2 did not increase mitochondrial iron depots in 636 mammalian cells and that mitochondria modulate their own iron import [71,72]

637 Several reports showed that in yeast the depletion of MRS3 and MRS4 did not change iron 638 accumulation within mitochondria or the activity of the iron sulphur proteins aconitase and 639 succinate dehydrogenase, except when the cells were deprived of iron $[28,30]$. The authors 640 concluded that when iron is available other iron transport systems are supplying the 641 mitochondrion and that MRS3 and MRS4 are only active when iron was low abundant [28,30]. 642 In contrast, in mammalian cells, the knock out of Mrfn1 and Mrfn2 abolished mitochondrial 643 iron import even when iron was provided to the medium[73]. Another study reported that the 644 depletion of MRS3 and MRS4 induced the yeast iron regulon and led to an increased iron 645 uptake into the cytoplasm and vacuole [73]. In rice shoots the depletion of the plant iron carrier 646 MIT caused an elevation of intracellular, but a reduction of mitochondrial, iron content [74].

647 It appears that the regulation of the intracellular iron distribution in $T$. brucei is similar to that 648 in yeast. Although, unlike yeast, T. brucei does not possess a vacuole, excess iron might be 649 stored within a yet unidentified depot and released when cytosolic concentrations are below a 650 certain threshold level [75]. It was previously suggested that this putative iron store could 651 support the parasite's growth for up to $48 \mathrm{~h}$ when iron is absent from the culture medium or 652 when iron import to the cytoplasm is blocked $[14,76,77]$.

653 Taken together with the phylogenetic and the sequence analysis, our results suggest that $654 \mathrm{TbMCP} 17$ acts as a mitochondrial iron carrier in $T$. brucei. We further propose that this carrier 655 is particularly relevant for mitochondrial iron transport when iron is not readily available from 656 the environment. 
658

659 [1] J. Rodgers, Human African trypanosomiasis, chemotherapy and CNS disease, J.

660

661

662

663

664

665

666

667

668

669

670

671

672

673

674

675

676

677

678

679

680

681

682 Neuroimmunol. (2009). doi:10.1016/j.jneuroim.2009.02.007.

[2] M.P. Barrett, R.J. Burchmore, A. Stich, J.O. Lazzari, A.C. Frasch, J.J. Cazzulo, S. Krishna, The trypanosomiases, Lancet. (2003). doi:10.1016/S0140-6736(03)146946.

[3] D. Moreira, P. López-García, K. Vickerman, An updated view of kinetoplastid phylogeny using environmental sequences and a closer outgroup: Proposal for a new classification of the class Kinetoplastea, Int. J. Syst. Evol. Microbiol. (2004). doi:10.1099/ijs.0.63081-0.

[4] R. Sharma, E. Gluenz, L. Peacock, W. Gibson, K. Gull, M. Carrington, The heart of darkness : growth and form of Trypanosoma brucei in the tsetse fly, 25 (2009) $517-$ 524. doi:10.1016/j.pt.2009.08.001.

[5] A.G.M. Tielens, J.J. Van Hellemond, Differences in energy metabolism between Trypanosomatidae, Parasitol. Today. 14 (1998) 265-271. doi:10.1016/S01694758(98)01263-0.

[6] P.A.M. Michels, F. Bringaud, M. Herman, V. Hannaert, Metabolic functions of glycosomes in trypanosomatids, Biochim. Biophys. Acta - Mol. Cell Res. 1763 (2006) 1463-1477. doi:10.1016/j.bbamcr.2006.08.019.

[7] P.A.M. Michels, V. Hannaert, F. Bringaud, Metabolic aspects of glycosomes in Trypanosomatidae - New data and views, Parasitol. Today. 16 (2000) 482-489. doi:10.1016/S0169-4758(00)01810-X.

[8] V. Coustou, S. Besteiro, M. Biran, P. Diolez, V. Bouchaud, P. Voisin, P.A.M. Michels, P. Canioni, T. Baltz, F. Bringaud, ATP generation in the Trypanosoma brucei procyclic form: cytosolic substrate level is essential, but not oxidative 

doi:10.1074/jbc.M307872200.

[9] A.G.M. Tielens, New functions for parts of the krebs cycle in procyclic, Biochemistry. 280 (2005) 12451-12460. doi:10.1074/jbc.M412447200.

[10] S. Besteiro, M.P. Barrett, L. Rivière, F. Bringaud, Energy generation in insect stages of Trypanosoma brucei: Metabolism in flux, Trends Parasitol. 21 (2005) 185-191. doi:10.1016/j.pt.2005.02.008.

690

[11] Z. Verner, S. Basu, C. Benz, S. Dixit, E. Dobáková, D. Faktorová, H. Hashimi, E. Horáková, Z. Huang, Z. Paris, P. Peña-Diaz, L. Ridlon, J. Týč, D. Wildridge, A. Zíková, J. Lukeš, Malleable mitochondrion of Trypanosoma brucei, Int. Rev. Cell Mol. Biol. 315 (2015) 73-151. doi:10.1016/bs.ircmb.2014.11.001.

[12] S. Basu, E. Horáková, J. Luke, Biochim. Biophys. Acta Iron-associated biology of Trypanosoma brucei, 1860 (2016) 363-370. doi:10.1016/j.bbagen.2015.10.027.

[13] D. Steverding, The transferrin receptor of Trypanosoma brucei, Parasitol. Int. (2000). doi:10.1016/S1383-5769(99)00018-5.

[14] M.C. Taylor, A.P. Mclatchie, J.M. Kelly, Evidence that transport of iron from the lysosome to the cytosol in African trypanosomes is mediated by a mucolipin orthologue, 89 (2013) 420-432. doi:10.1111/mmi.12285.

[15] B. Vanhollebeke, G. De Muylder, M.J. Nielsen, A. Pays, P. Tebabi, M. Dieu, M. Raes, S.K. Moestrup, E. Pays, A haptoglobin-hemoglobin receptor conveys innate immunity to Trypanosoma brucei in humans, Science (2008). doi:10.1126/science. 1156296.

[16] J. Mach, J. Tachezy, R. Sutak, Efficient Iron Uptake via a Reductive Mechanism in Procyclic Trypanosoma brucei, J. Parasitol. (2013). doi:10.1645/ge-3237.1.

[17] J. Lukeš, S. Basu, Fe/S protein biogenesis in trypanosomes - A review, Biochim. 

Biophys. Acta - Mol. Cell Res. (2015). doi:10.1016/j.bbamcr.2014.08.015.

[18] M. Dormeyer, R. Schoneck, G.A. Dittmar, R.L. Krauth-Siegel, Cloning, sequencing and expression of ribonucleotide reductase R2 from Trypanosoma brucei, FEBS Lett. (1997). doi:S0014-5793(97)01036-3 [pii].

[19] A. Hofer, P.P. Schmidt, A. Gräslund, L. Thelander, Cloning and characterization of the R1 and R2 subunits of ribonucleotide reductase from Trypanosoma brucei, Proc. Natl. Acad. Sci. U. S. A. (1997). doi:10.1073/pnas.94.13.6959.

[20] N. Le Trant, S.R. Meshnick, K. Kitchener, J.W. Eaton, A. Cerami, Iron-containing superoxide dismutase from Crithidia fasciculata. Purification, characterization, and similarity to Leishmanial and trypanosomal enzymes., J. Biol. Chem. (1983).

[21] A.B. Clarkson, E.J. Bienen, G. Pollakis, R.W. Grady, Respiration of bloodstream forms of the parasite Trypanosoma brucei brucei is dependent on a plant-like alternative oxidase, J. Biol. Chem. (1989).

[22] A. Tsuda, W.H. Witola, K. Ohashi, M. Onuma, Expression of alternative oxidase inhibits programmed cell death-like phenomenon in bloodstream form of Trypanosoma brucei rhodesiense, Parasitol. Int. (2005). doi:10.1016/j.parint.2005.06.007.

[23] A. Tsuda, W.H. Witola, S. Konnai, K. Ohashi, M. Onuma, The effect of TAO expression on PCD-like phenomenon development and drug resistance in Trypanosoma brucei, Parasitol. Int. (2006). doi:10.1016/j.parint.2006.01.001.

[24] P. Overath, J. Czichos, C. Haas, The effect of citrate/cis-aconitate on oxidative metabolism during transformation of Trypanosoma brucei, Eur. J. Biochem. (1986). doi:10.1111/j.1432-1033.1986.tb09955.x.

[25] M.C. Taylor, J.M. Kelly, Iron metabolism in trypanosomatids, and its crucial role in infection, Parasitology. 137 (2010) 899-917. doi:10.1017/S0031182009991880. 
733

734

735

736

737

738

739

740

741

742

743

744

745

746

747

748

749

750

751

752

753

754

755

756

757

[26] A. Tangerås, T. Flatmark, D. Bäckström, A. Ehrenberg, Mitochondrial iron not bound in heme and iron-sulfur centers. Estimation, compartmentation and redox state, BBA - Bioenerg. (1980). doi:10.1016/0005-2728(80)90035-3.

[27] T.A. Rouault, W.H. Tong, Iron-sulphur cluster biogenesis and mitochondrial iron homeostasis, Nat. Rev. Mol. Cell Biol. (2005). doi:10.1038/nrm1620.

[28] F. Foury, T. Roganti, Deletion of the mitochondrial carrier genes MRS3 and MRS4 suppresses mitochondrial iron accumulation in a yeast frataxin-deficient strain, J. Biol. Chem. 277 (2002) 24475-24483. doi:10.1074/jbc.M111789200.

[29] M.R. Felice, I. De Domenico, L. Li, D.M. Ward, B. Bartok, G. Musci, J. Kaplan, Post-transcriptional regulation of the yeast high affinity iron transport system., J. Biol. Chem. (2005). doi:10.1074/jbc.M414663200.

[30] U. Mühlenhoff, J.A. Stadler, N. Richhardt, A. Seubert, T. Eickhorst, R.J. Schweyen, R. Lill, G. Wiesenberger, A Specific Role of the Yeast Mitochondrial Carriers Mrs3/4p in Mitochondrial Iron Acquisition under Iron-limiting Conditions, J. Biol. Chem. 278 (2003) 40612-40620. doi:10.1074/jbc.M307847200.

[31] G.C. Shaw, J.J. Cope, L. Li, K. Corson, C. Hersey, G.E. Ackermann, B. Gwynn, A.J. Lambert, R.A. Wingert, D. Traver, N.S. Trede, B.A. Barut, Y. Zhou, E. Minet, A. Donovan, A. Brownlie, R. Balzan, M.J. Weiss, L.L. Peters, J. Kaplan, L.I. Zon, B.H. Paw, Mitoferrin is essential for erythroid iron assimilation, Nature. (2006). doi:10.1038/nature04512.

[32] H. Aquila, T.A. Link, M. Klingenberg, Solute carriers involved in energy transfer of mitochondria form a homologous protein family, FEBS Lett. (1987). doi:10.1016/0014-5793(87)81546-6.

[33] F. Palmieri, The mitochondrial transporter family (SLC25): Physiological and pathological implications, Pflugers Arch. Eur. J. Physiol. (2004). 
759 [34] F. Palmieri, Diseases caused by defects of mitochondrial carriers: A review, Biochim. Biophys. Acta - Bioenerg. (2008). doi:10.1016/j.bbabio.2008.03.008.

[35] E.R.S. Kunji, A.J. Robinson, The conserved substrate binding site of mitochondrial carriers, Biochim. Biophys. Acta - Bioenerg. (2006). doi:10.1016/j.bbabio.2006.03.021.

[36] C. Colasante, P. Peña Diaz, C. Clayton, F. Voncken, Mitochondrial carrier family inventory of Trypanosoma brucei brucei: Identification, expression and subcellular localisation, Mol. Biochem. Parasitol. 167 (2009) 104-117. doi:10.1016/j.molbiopara.2009.05.004. doi:10.1016/j.molbiopara.2018.03.003. McWilliam, M. Remmert, J. Söding, J.D. Thompson, D.G. Higgins, Fast, scalable generation of high-quality protein multiple sequence alignments using Clustal 

Omega., Mol. Syst. Biol. 7 (2011) 539. doi:10.1038/msb.2011.75.

784 [41] W. Li, A. Cowley, M. Uludag, T. Gur, H. McWilliam, S. Squizzato, Y.M. Park, N.

785

786

Buso, R. Lopez, The EMBL-EBI bioinformatics web and programmatic tools framework., Nucleic Acids Res. 43 (2015) W580-4. doi:10.1093/nar/gkv279.

[42] S. Kumar, G. Stecher, K. Tamura, MEGA7: Molecular Evolutionary Genetics Analysis Version 7.0 for Bigger Datasets., Mol. Biol. Evol. 33 (2016) 1870-4. doi:10.1093/molbev/msw054.

[43] N. Saitou, M. Nei, The neighbor-joining method: a new method for reconstructing phylogenetic trees., Mol. Biol. Evol. 4 (1987) 406-25.

[44] S. Biebinger, S. Rettenmaier, J. Flaspohler, C. Hartmann, J. Peña-Diaz, L. Elizabeth Wirtz, H.R. Hotz, J.D. Barry, C. Clayton, The PARP promoter of Trypanosoma brucei is developmentally regulated in a chromosomal context, Nucleic Acids Res. 24 (1996) 1202-1211. doi:10.1093/nar/24.7.1202.

[45] E.M. Froschauer, R.J. Schweyen, G. Wiesenberger, The yeast mitochondrial carrier proteins $\mathrm{Mrs} 3 \mathrm{p} / \mathrm{Mrs} 4 \mathrm{p}$ mediate iron transport across the inner mitochondrial membrane., Biochim. Biophys. Acta. 1788 (2009) 1044-50. doi:10.1016/j.bbamem.2009.03.004.

[46] R.D. Gietz, R.A. Woods, Transformation of yeast by lithium acetate/single-stranded carrier DNA/polyethylene glycol method, Methods Enzymol. 350 (2002) 87-96. doi:10.1016/S0076-6879(02)50957-5.

[47] E. Garí, L. Piedrafita, M. Aldea, E. Herrero, A set of vectors with a tetracyclineregulatable promoter system for modulated gene expression in Saccharomyces cervisiae, Yeast. (1997). doi:10.1002/(SICI)1097-0061(199707)13:9<837::AIDYEA145>3.0.CO;2-T.

[48] F. Voncken, F. Gao, C. Wadforth, M. Harley, C. Colasante, The Phosphoarginine 

Energy-Buffering System of Trypanosoma brucei Involves Multiple Arginine Kinase Isoforms with Different Subcellular Locations, PLoS One. (2013). doi:10.1371/journal.pone.0065908.

[49] S. Krieger, W. Schwarz, M.R. Ariyanayagam, A.H. Fairlamb, R.L. Krauth-Siegel, C. Clayton, Trypanosomes lacking trypanothione reductase are avirulent and show increased sensitivity to oxidative stress., Mol. Microbiol. 35 (2000) 542-52.

[50] F. Voncken, J.J. Van Hellemond, I. Pfisterer, A. Maier, S. Hillmer, C. Clayton, Depletion of GIM5 causes cellular fragility, a decreased glycosome number, and reduced levels of ether-linked phospholipids in trypanosomes, J. Biol. Chem. 278 (2003) 35299-35310. doi:10.1074/jbc.M301811200.

[51] V. Bellofatto, J.B. Palenchar, RNA interference as a genetic tool in trypanosomes, Methods Mol. Biol. 442 (2008) 83-94. doi:10.1007/978-1-59745-191-8-7.

[52] A. Schneider, D. Bursać, T. Lithgow, The direct route: a simplified pathway for protein import into the mitochondrion of trypanosomes, Trends Cell Biol. 18 (2008) 12-18. doi:10.1016/j.tcb.2007.09.009.

[53] J. Riemer, H. Hoepken, H. Czerwinska, S.R. Robinson, R. Dringen, Colorimetric ferrozine-based assay for the quantitation of iron in cultured cells, 331 (2004) 370375. doi:10.1016/j.ab.2004.03.049.

[54] F.Y. Li, B. Leibiger, I. Leibiger, C. Larsson, Characterization of a putative murine mitochondrial transporter homology of hMRS3/4, Mamm. Genome. (2002). doi:10.1007/s00335-001-2094-y.

[55] F. Palmieri, The mitochondrial transporter family (SLC25): Physiological and pathological implications, Pflugers Arch. Eur. J. Physiol. 447 (2004) 689-709. doi:10.1007/s00424-003-1099-7.

[56] H. Aquila, D. Misra, M. Eulitz, M. Klingenberg, Complete amino acid sequence of 

the ADP/ATP carrier from beef heart mitochondria., Hoppe-Seyler's Zeitschrift Für Physiol. Chemie. 363 (1982) 345-9. http://www.ncbi.nlm.nih.gov/pubmed/7076130.

[57] M. Saraste, J.E. Walker, Internal sequence repeats and the path of polypeptide in mitochondrial ADP/ATP translocase, FEBS Lett. 144 (1982) 250-254.

[58] A.J. Robinson, E.R.S. Kunji, Mitochondrial carriers in the cytoplasmic state have a common substrate binding site, Proc. Natl. Acad. Sci. (2006).

[59] M. Gutiérrez-Aguilar, C.P. Baines, Physiological and pathological roles of doi:10.1073/pnas.0509994103.

[60] V. Zara, A. Ferramosca, P. Robitaille-Foucher, F. Palmieri, J.C. Young, Mitochondrial carrier protein biogenesis: Role of the chaperones Hsc70 and Hsp90, Biochem. J. (2009). doi:10.1042/BJ20082270.

[61] A. Ferramosca, V. Zara, Biogenesis of mitochondrial carrier proteins: Molecular

[62] B. Stijlemans, A. Beschin, S. Magez, J.A. Van Ginderachter, P. De Baetselier, Iron 850

[64] K.E.J. Tripodi, S.M. Menendez Bravo, J.A. Cricco, Role of heme and heme-proteins

[65] V. Hannaert, E. Saavedra, F. Duffieux, J.-P. Szikora, D.J. Rigden, P.A.M. Michels, 
parasites., Proc. Natl. Acad. Sci. U. S. A. 100 (2003) 1067-71.

859 doi:10.1073/pnas.0335769100.

860 [66] P.J. Keeling, Diversity and evolutionary history of plastids and their hosts, Am. J. Bot. 91 (2004) 1481-1493. doi:10.3732/ajb.91.10.1481.

862

863

864

865

866

867

868

869

870

[67] P.J. Keeling, J.D. Palmer, Horizontal gene transfer in eukaryotic evolution, Nat. Rev. Genet. 9 (2008) 605-618. doi:10.1038/nrg2386.

[68] F.R. Opperdoes, P.A.M. Michels, Horizontal gene transfer in trypanosomatids., Trends Parasitol. 23 (2007) 470-476. doi:10.1016/j.pt.2007.08.002.

[69] M.D. Fleming, D.R. Campagna, J.N. Haslett, C.C. Trenor, N.C. Andrews, A mutation in a mitochondrial transmembrane protein is responsible for the pleiotropic hematological and skeletal phenotype of flexed-tail (f/f) mice, Genes Dev. 15 (2001) 652-657. doi:10.1101/gad.873001.

[70] X. Ye, J. Xu, C. Cheng, G. Yin, L. Zeng, C. Ji, S. Gu, Y. Xie, Y. Mao, Isolation and characterization of a novel human putative anemia-related gene homologous to mouse sideroflexin, Biochem. Genet. (2003). doi:10.1023/A:1022026001114.

[71] D.R. Richardson, D.J.R. Lane, E.M. Becker, M.L.H. Huang, M. Whitnall, Y.S. Rahmanto, A.D. Sheftel, P. Ponka, Mitochondrial iron trafficking and the integration of iron metabolism between the mitochondrion and cytosol, Proc. Natl. Acad. Sci. U. S. A. (2010). doi:10.1073/pnas.0912925107.

[72] P.N. Paradkar, K.B. Zumbrennen, B.H. Paw, D.M. Ward, J. Kaplan, Regulation of mitochondrial iron import through differential turnover of mitoferrin 1 and mitoferrin 2, Mol. Cell. Biol. (2009). doi:10.1128/mcb.01685-08.

[73] L. Li, J. Kaplan, A mitochondrial-vacuolar signaling pathway in yeast that affects iron and copper metabolism, J. Biol. Chem. 279 (2004) 33653-33661. doi:10.1074/jbc.M403146200. 
883

884

885

886

887

888

889

890

891

892

893

894

895

896

897

898

899

900

901

902

903

904

905

906

907

[74] K. Bashir, Y. Ishimaru, H. Shimo, S. Nagasaka, M. Fujimoto, H. Takanashi, N. Tsutsumi, G. An, H. Nakanishi, N.K. Nishizawa, The rice mitochondrial iron transporter is essential for plant growth, Nat. Commun. 2 (2011) 322-327. doi:10.1038/ncomms1326.

[75] B. Stijlemans, A. Vankrunkelsven, L. Brys, S. Magez, P. De Baetselier, Role of iron homeostasis in trypanosomiasis-associated anemia, Immunobiology. (2008). doi:10.1016/j.imbio.2008.07.023.

[76] D. Steverding, Bloodstream forms of Trypanosoma brucei require only small amounts of iron for growth, Parasitol. Res. (1997). doi:10.1007/s004360050357.

[77] H.G.A.M. Van Luenen, R. Kieft, R. Mußmann, M. Engstler, B. Ter Riet, P. Borst, Trypanosomes change their transferrin receptor expression to allow effective uptake of host transferrin, Mol. Microbiol. (2005). doi:10.1111/j.1365-2958.2005.04831.x.

\section{AKNOWLEDGEMENT}

We thank Christine Clayton for proof-reading the manuscript.

This work was funded by the BBSRC, grant number BB/G00448X/1.

\section{CONFLICT OF INTEREST}

The authors declare that they have no conflicts of interest with the contents of this article.

\section{AUTHOR CONTRIBUTION}

FZ, CC and FV designed all the experiments presented in this paper and experiments were conducted by FZ. All authors reviewed the results and contributed equally to the drafting and correction of the manuscript as well as to the design of the figures. The final version of the manuscript was approved by all authors. 